\title{
The SWIRE-VVDS-CFHTLS surveys: stellar mass assembly over the last 10 Gyr. Evidence for a major build up of the red sequence between $z=2$ and $z=1^{\star}$
}

\author{
S. Arnouts ${ }^{1,2}$, C. J. Walcher ${ }^{2}$, O. Le Fèvre ${ }^{2}$, G. Zamorani ${ }^{3}$, O. Ilbert ${ }^{4}$, V. Le Brun ${ }^{2}$, L. Pozzetti ${ }^{3}$, S. Bardelli ${ }^{3}$, \\ L. Tresse ${ }^{2}$, E. Zucca ${ }^{3}$, S. Charlot ${ }^{5}$, F. Lamareille ${ }^{6}$, H. J. McCracken ${ }^{5}$, M. Bolzonella ${ }^{3}$, A. Iovino ${ }^{7}$, C. Lonsdale ${ }^{8}$, \\ M. Polletta ${ }^{8}$, J. Surace ${ }^{9}$, D. Bottini ${ }^{10}$, B. Garilli ${ }^{10}$, D. Maccagni ${ }^{10}$, J. P. Picat ${ }^{6}$, R. Scaramella ${ }^{3}$, M. Scodeggio ${ }^{10}$,

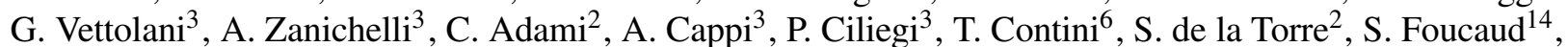 \\ P. Franzetti ${ }^{10}$, I. Gavignaud ${ }^{15}$, L. Guzzo ${ }^{7}$, B. Marano ${ }^{3}$, C. Marinoni ${ }^{13}$, A. Mazure ${ }^{2}$, B. Meneux ${ }^{7}$, R. Merighi ${ }^{3}$, \\ S. Paltani ${ }^{12}$, R. Pellò ${ }^{6}$, A. Pollo ${ }^{2}$, M. Radovich ${ }^{11}$, S. Temporin ${ }^{7}$, and D. Vergani ${ }^{10}$ \\ (Affiliations can be found after the references)
}

Received 12 April 2007 / Accepted 18 September 2007

\begin{abstract}
We present an analysis of the stellar mass growth over the last $10 \mathrm{Gyr}(z \leq 2)$ using a unique large sample of galaxies selected at $3.6 \mu \mathrm{m}$. We have assembled accurate photometric and spectroscopic redshifts for $\sim 21200$ and 1500 galaxies, respectively, with $F(3.6 \mu \mathrm{m}) \geq 9.0 \mu \mathrm{Jy}$ by combining data from Spitzer-SWIRE IRAC, the VIMOS VLT Deep Survey (VVDS), UKIDSS and very deep optical CFHTLS photometry. We split our sample into quiescent (red) and active (blue) galaxies on the basis of an SED fitting procedure that we have compared with the strong rest-frame color bimodality $\left(N U V-r^{\prime}\right)_{\mathrm{ABS}}$. The present sample contains $\sim 4400$ quiescent galaxies. Our measurements of the K-rest frame luminosity function and luminosity density evolution support the idea that a large fraction of galaxies is already assembled at $z \sim 1.2$, with almost $80 \%$ and $50 \%$ of the active and quiescent populations already in place, respectively.

Based on the analysis of the evolution of the stellar mass-to-light ratio (in $K$-band) for the spectroscopic sub-sample, we derive the stellar mass density for the entire sample. We find that the global evolution of the stellar mass density is well reproduced by the star formation rate derived from UV based measurements when an appropriate dust correction is applied, which supports the idea of an initial mass function that is on average universal.

Over the last $8 \mathrm{Gyr}(z \leq 1.2)$ we observe that the stellar mass density of the active population shows a modest mass growth rate $(\dot{\rho} \sim$ $\left.0.005( \pm 0.005) M_{\odot} / \mathrm{Mpc}^{3} / \mathrm{yr}\right)$, consistent with a constant stellar mass density, $\rho_{\star}^{\text {active }} \sim 3.1 \times 10^{8} M_{\odot} / \mathrm{Mpc}^{3}$. In contrast, an increase by a factor of $\sim 2$ for the quiescent population over the same timescale is observed. As a consequence, the growth of the stellar mass in the quiescent population must be due to the shutoff of star formation in active galaxies that migrate into the quiescent population. We estimate this stellar mass flux to be $\dot{\rho}_{A \rightarrow Q} \sim 0.017( \pm 0.004) M_{\odot} / \mathrm{Mpc}^{3} / \mathrm{yr}$, which balances the major fraction of new stars born according to our best SFR estimate $\left(\dot{\rho}=0.025( \pm 0.003) M_{\odot} / \mathrm{Mpc}^{3} / \mathrm{yr}\right)$.

From $z=2$ to $z=1.2$, we observe a major build-up of the quiescent population with an increase by a factor of $\sim 10$ in stellar mass (a mass growth rate of $\sim 0.063 M_{\odot} / \mathrm{Mpc}^{3} / \mathrm{yr}$ ). This rapid evolution suggests that we are observing the epoch when, for the first time in the history of the universe, an increasing fraction of galaxies end their star formation activity and start to build up the red sequence.
\end{abstract}

Key words. galaxies: luminosity function, mass function - galaxies: formation

\section{Introduction}

The strong decline of the cosmic star formation rate (SFR) since $z \sim 1$ is now well established (Schiminovich et al. 2005; Hopkins et al. 2006), and has been shown to be accompanied by a decrease of faint star forming galaxies and the decline of

$\star$ Based on data obtained with the European southern observatory Very Large Telescope, Paranal, Chile, program 070A-9007(A) and on observations obtained with MegaPrime/MegaCam, a joint project of CFHT and CEA/DAPNIA, at the Canada-France-Hawaii Telescope (CFHT) which is operated by the National Research Council (NRC) of Canada, the Institut National des Science de l'Univers of the Centre National de la Recherche Scientifique (CNRS) of France, and the University of Hawaii. This work is based in part on data products produced at TERAPIX and the Canadian Astronomy Data Centre as part of the Canada-France-Hawaii Telescope Legacy Survey, a collaborative project of NRC and CNRS and on data obtained as part of the UKIRT Infrared Deep Sky Survey. luminous ultra-violet galaxies (Lilly et al. 1996; Arnouts et al. 2005). One fundamental question is to understand the link between the global decline of the star formation rate and the evolution of the mass assembly. Inspection of the specific star formation rate (SFR per unit of stellar mass) in individual galaxies reveals that the preferred site of star formation activity has migrated from massive systems at high $z$ to low mass systems at low $-z$. This is usually referred to as the downsizing effect (Cowie et al. 1996; Juneau et al. 2005). Evidences of such an effect have been seen in the fundamental plane relation of elliptical galaxies (Treu et al. 2005) as well as in the analysis of spectra of local galaxies (Heavens et al. 2004; Kauffmann et al. 2003) where both analyses show that massive galaxies have formed their stars earlier than less massive ones. Clustering properties of star-forming galaxies at high and low redshifts reveal that the bulk of star formation activity has migrated from massive dark matter halos (DMH) at high $z$ to low mass DMH at low $z$, 
generalizing the downsizing effect from stellar mass to the dark matter (Heinis et al. 2007).

While the $\Lambda \mathrm{CDM}$ hierarchical scenario successfully describes the clustering properties of galaxies on large scales (Mo \& White 1996; Springel et al. 2006), the quenching of star formation in massive systems is less well understood. It relies on the complex physics of baryons and there is not yet enough constraints on the mechanisms involved in the regulation of star formation. These mechanisms may act on galactic scales like AGN, Supernovae feedback (Benson et al. 2003; Croton et al. 2006) or on large scales via merging or gas heating in dense environments (Naganime et al. 2001; Yoshikawa et al. 2001). For instance, to reproduce the properties of local massive elliptical galaxies, de Lucia et al. (2006) show that the stars could form first at high redshift in sub-galactic units, while the galaxies would continue their stellar mass assembly through merging onto low redshift.

In this context, the most stringent constraint for the semianalytical models is the direct observation of the number density of massive galaxies at high redshift. Evidences of the existence of massive galaxies at high redshift are becoming numerous. An important population of EROs at $z \geq 1$ has been discovered (K20 survey: Cimatti et al. 2002), with roughly half of them being old stellar systems (Cimatti et al. 2004). They could be the already assembled progenitors of local massive ellipticals, as supported by their clustering signals and number density (Daddi et al. 2002; Arnouts 2003). The measures of the evolution of the stellar mass density from NIR surveys found that half of the stellar mass is already in place at $z \leq 1-1.5$ (Fontana et al. 2003, 2004; Pozzetti et al. 2003; Drory et al. 2004, 2005; Caputi et al. 2005) moving the formation epoch close to the peak of the cosmic SFR. The analysis of the galaxy mass function (GMF) at different redshifts provides additional information on how the process of mass assembly acts on different mass scales. Such analysis is becoming common among the recent surveys (Fontana et al. 2004, 2006; Drory et al. 2004, 2005; Bundy et al. 2005; Borch et al. 2006; Franceschini et al. 2006; Pozzetti et al. 2007). While the different studies do not necessarily agree with each other, it emerges that the most massive galaxies have undergone less evolution than less massive ones since $z \sim 1.0$ and 50 to $70 \%$ are already in place at such a redshift, revealing a faster assembly for the most massive systems.

An alternative approach to measure the density of massive galaxies at high redshift is to investigate the evolution of the local elliptical galaxies that dominate the massive end of the GMF (Bell et al. 2003; Baldry et al. 2004; Bundy et al. 2006; Cirasuolo et al. 2006). This has been done by following the redshift evolution of the red sequence, defined by the $(U-V)$ or $(U-B)$ colors, from today up to $z \sim 1$.1. Bell et al. (2004) and Faber et al. (2005) find that the density of red galaxies drops by a factor 4 or 2.5 respectively, suggesting that their stellar mass density roughly doubles, while at the same time the blue population shows little evolution. They interpret this evolution by the migration of blue galaxies that quenched their star formation and migrated into the red sequence. Bell et al. (2004) also reported a significant evolution of the most massive ellipticals. Because there is a shortage of massive blue galaxies able to produce those massive ellipticals, they introduce the idea of "dry" or purely stellar mergers (merging between red galaxies) as a possible scenario to produce these massive local ellipticals.

Analysis from the VVDS survey by Zucca et al. (2006), who define the early-type class based on SED fitting, report a more modest decline $(\sim 40 \%)$ in the number density of this population up to $z \sim 1.1$, which is also consistent with the analysis by
Ilbert et al. (2006b) based on a morphologically selected elliptical sample in the CDF South.

Similarly to VVDS results, Brown et al. (2007), who have selected ellipticals with same optical color criteria than Bell et al. (2004), in an area of $7 \mathrm{deg}^{2}$, do not observe any evolution of comoving density $\left(\Phi^{\star}\right)$ up to $z=1$ and according to the modest luminosity density increase $(\sim 36 \%)$, predicts that the stellar mass has roughly doubled since $z \sim 1$. Moreover, they observe a modest evolution of luminous ellipticals (with $L \geq 4 L^{\star}$ ) with $80 \%$ of their stellar mass in place at $z \sim 0.8$, suggesting that "dry" mergers should not play a dominant role in the evolution of ellipticals over the last $8 \mathrm{Gyr}$.

Complementary analysis by Bundy et al. (2006) find signatures of a downsizing effect with quenching affecting first the massive galaxies and then moving to lower mass systems. This can be interpreted as an anti-hierarchical process with massive early types ending first their mass assembly while the less massive ones are still assembling their mass (see also Cimatti et al. 2006). If such a process is confirmed, it would be a strong observational constraint for the models of galaxy formation within the standard paradigm.

A large fraction of the surveys discussed above are limited to $z \leq 1-1.2$, due to optical selection, while the deepest surveys are still covering modest sizes in particular when the sample is splitted by galaxy types. The impact of cosmic variance on results coming from small fields remains a major problem. Even in the three fields covered by COMBO17, it still appears to play a significant role (Bell et al. 2004; Somerville et al. 2004). The selection of the samples varies from optical to IR band and the stellar mass is estimated from various approaches including SED fitting, rest-frame colors, with or without infrared information, introducing significant dispersions in the final estimates. Additionally, the selection of elliptical galaxies based on optical colors has been found to show a significant contamination by Sey2 AGN and star forming galaxies up to $40 \%$ (Franzetti et al. 2007).

In this paper we explore the redshift evolution of the stellar mass assembly for a unique large sample selected in the observed Mid-Infrared, via the analysis of the K-rest luminosity functions, the luminosity density and the evolution of the mass to light ratio. We take advantage of a native IRAC selection that allows us to detect massive red objects up to $z \sim 2$, and we isolate a large sample of red/quiescent galaxies according to their low level of star formation activity. We then discuss implications for the stellar mass assembly of the active and quiescent populations. Throughout the paper we adopt the concordance cosmology $\Omega_{\mathrm{m}}=0.3$ and $\Omega_{\Lambda}=0.7$ and $H_{0}=70 \mathrm{~km} \mathrm{~s}^{-1} \mathrm{Mpc}^{-1}$. All magnitudes are in the $\mathrm{AB}$ system, and for convenience we adopt a Salpeter IMF unless otherwise specified.

\section{The data}

In this work we make use of the large amount of data collected in the VVDS-0226-04 field. The present sample is based on a $3.6 \mu \mathrm{m}$ flux limited sample from the SWIRE survey which overlaps the deep multi-colour imaging survey from the CFHTLS. The common area between the two surveys corresponds to 0.85 square degree. We complete this dataset by including the deep spectro-photometric data from the VVDS survey and the infrared data from the UKIDSS survey. 


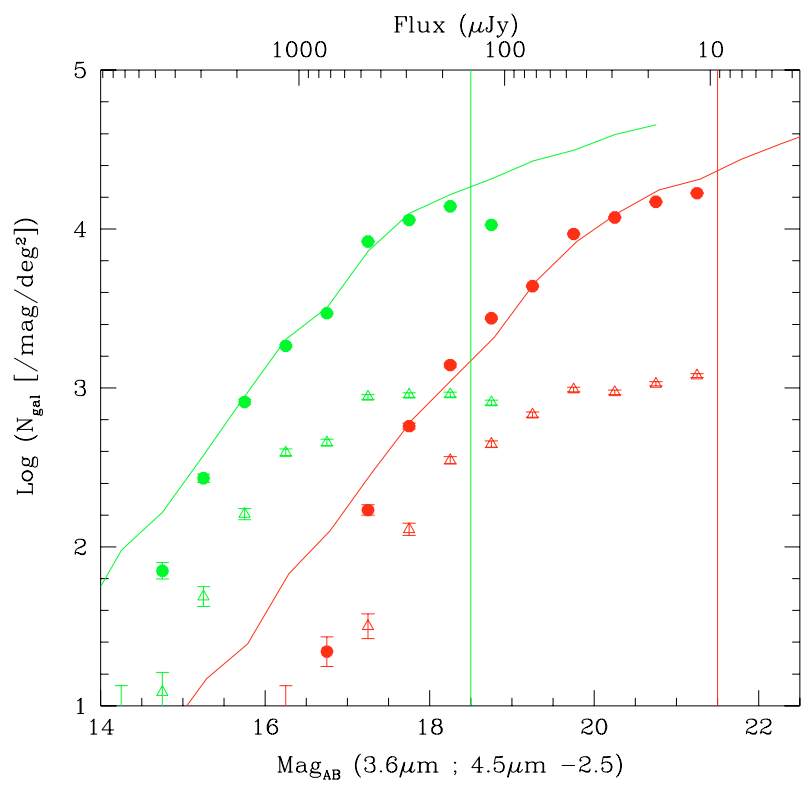

Fig. 1. Stars (triangles) and Galaxy (circles) number counts for the $3.6 \mu \mathrm{m}$ (red symbols) and $4.5 \mu \mathrm{m}$ (green symbols) samples. Solid lines show the IRAC number counts from Fazio et al. (2005).

\subsection{The SWIRE sample}

The present sample corresponds to a $3.6 \mu \mathrm{m}$ flux limited sample with $F(3.6 \mu \mathrm{m}) \geq 9 \mu \mathrm{Jy}$ ( or $m_{\mathrm{AB}}(3.6) \leq 21.5$ ), based on the SWIRE survey (Lonsdale et al. 2003). The SWIRE photometry is based on the band-merged catalog including 3.6, 4.5, 5.6, $8.0 \mu \mathrm{m}$ and $24 \mu \mathrm{m}$ passband (Surace et al. 2005). with a typical $5 \sigma$ depth of $5.0,9.0,43,40$ and $311 \mu \mathrm{Jy}$ respectively We use the flux measurements derived in 3 arcsec apertures for faint sources as suggested by Surace et al. (2005), while we adopt the adaptive apertures (Kron magnitude; Bertin \& Arnouts 1996) for the bright sources $\left(m_{\mathrm{AB}}(3.6) \leq 19.5\right)$. In Fig. 1, we show the IRAC 3.6 and $4.5 \mu \mathrm{m}$ number counts. When comparing with the number counts from Fazio et al. (2005), the $3.6 \mu \mathrm{m}$ galaxy number counts are $\sim 80 \%$ complete at $9 \mu \mathrm{Jy}$. At this depth within the common area with CFHTLS, we have 25500 sources.

\subsection{The CFHTLS data}

The deep multi-colour photometry $\left(u^{*} g^{\prime} r^{\prime} i^{\prime} z^{\prime}\right)$ from the CanadaFrance-Hawaii Telescope Legacy Survey is based on the T0003 release (CFHTLS-D1). These data cover one square degree, with sub-arcsecond seeing in all bands and reach the limiting magnitudes (corresponding to 50\% completeness) of 26.6, 26.5, 26.0, 26.0 and 25.2 in $u^{*}, g^{\prime}, r^{\prime}, i^{\prime}, z^{\prime}$ respectively. A full description of the data will be presented in a forthcoming paper by Mc Cracken et al. (in prep.).

Thanks to the very deep optical data, almost all the IRAC sources have an optical counterpart, except a negligible fraction $(\sim 0.5 \%)$, due to unmatched positions within $1.5 \mathrm{arcsec}$.

\subsection{The VVDS data}

The VIMOS VLT Deep Survey consists of deep photometry and spectroscopy (Le Fèvre et al. 2005):

- Deep $B, V, R, I$ imaging with a depth (50\% completeness) of 26.5, 26.2, 25.9, 25.0, respectively (Mc Cracken et al. 2003). In addition, $\mathrm{J}$ and $\mathrm{K}$ observations with NTT-SOFI have been

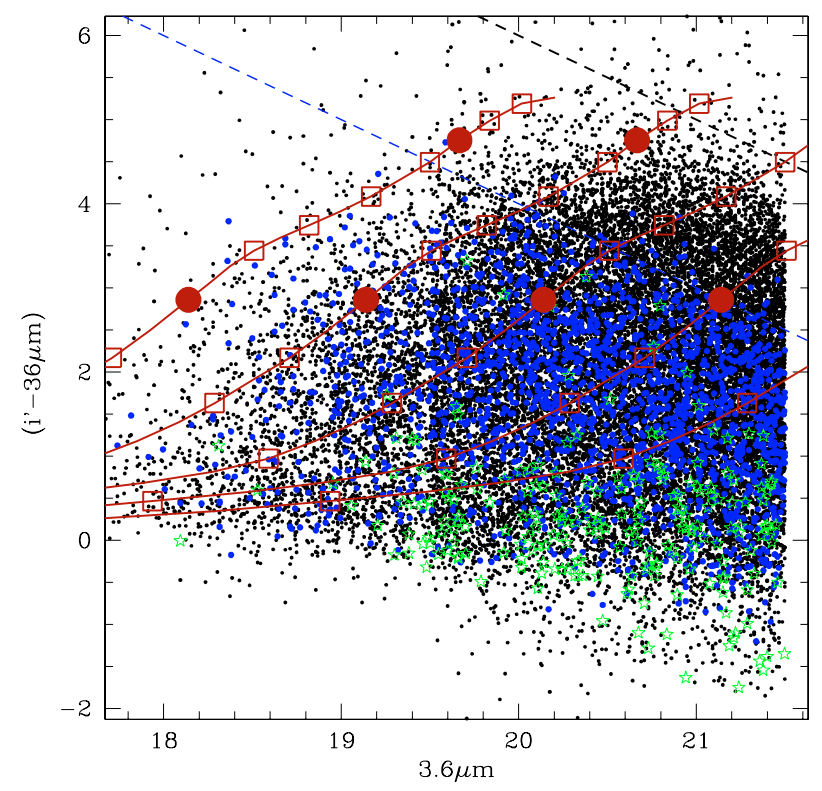

Fig. 2. Color-magnitude distribution with $(i-3.6 \mu \mathrm{m})$ vs. $3.6 \mu \mathrm{m}$. We show the spectroscopic sample for galaxies (filled blue symbols) and for stars (green stars symbols). The spectroscopic flux limit $(I \leq 24)$ and the $5 \sigma$ detection limit for the CFHTLS $\left(i^{\prime} \sim 26\right)$ are shown as dashed lines (lower and upper line respectively). The solid lines show the behaviour of an elliptical galaxy (with $\tau=0.1 \mathrm{Gyr}$ and $z_{\text {form }}=6$ ) moving from $z=2.5$ to $z=0$ (from red to blue color) and for different absolute $\mathrm{K}$ magnitudes between $K_{\mathrm{ABS}}=-25$ (left solid line) and $K_{\mathrm{ABS}}=-21$ (right solid line). The large circles denote the redshifts: $z=1.0,2.0$, while open squares are spaced by $\delta z=0.2$.

obtained at the depth (50\% completeness) of 24.2 and 23.8 , respectively over $172 \operatorname{arcmin}^{2}$ (Iovino et al. 2005).

- The first epoch VVDS spectroscopic sample is based on a randomly selected sample of $\sim 9000$ sources in the magnitude range $17 \leq I \leq 24.0$ (Le Fèvre et al. 2004). The spectroscopic area overlapping the SWIRE-CFHTLS data is $0.42 \mathrm{deg}^{2}$ and provides $\sim 1500$ secure spectra for sources with $m_{\mathrm{AB}}(3.6 \mu \mathrm{m}) \leq 21.5$.

\subsection{The UKIDSS data}

Finally, we complete our dataset with the $\mathrm{J}$ and $\mathrm{K}$ photometry from the UKIDSS Ultra Deep Survey (Lawrence et al. 2006) based on the DR1 release (Warren et al. 2007). These data reach a depth ( $5 \sigma$ limits) of 22.5 and 22.0 in $\mathrm{J}$ and $\mathrm{K}$, respectively. The overlap with SWIRE-CFHTLS is 0.55 and $0.76 \mathrm{deg}^{2}$ for $J$ and $K$ bands, respectively. We match the UDS sources with the optical data within a 1 arcsec radius. Within the common areas, $89 \%$ (or 93\%) of the 3.6 selected sources have a J (or K) flux measurement.

\subsection{The combined sample}

In Fig. 2, we show the $(i-3.6 \mu \mathrm{m})$ vs. $3.6 \mu \mathrm{m}$ color-magnitude diagram for the whole sample (small dots). The galaxies and stars from the spectroscopic sample are shown as blue and green symbols respectively. We plot the cut-offs introduced in the spectroscopic sample due to the magnitude limit, $I_{\mathrm{AB}} \leq 24$ (lower dashed line) and in the CFHTLS by adopting the $5 \sigma$ detection limit, $i^{\prime} \sim 26$ (upper dashed line). The evolutionary tracks of an elliptical galaxy, formed at $z=6$ and with an e-folding parameter $\tau=0.1$ Gyr (using the PEGASE model; Fioc et al. 1997) 


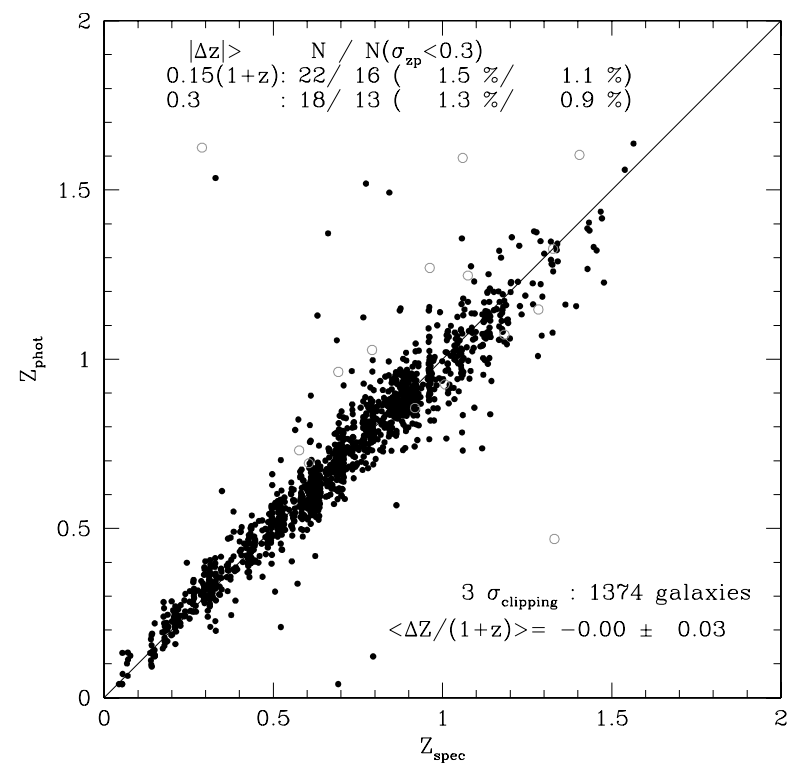

Fig. 3. Photometric vs. spectroscopic redshift comparison, for $\sim 1400$ secure VVDS spectroscopic redshifts. Open symbols refer to objects with internal photo- $z$ uncertainties $\sigma\left(z_{\text {phot }}\right) \geq 0.3$.

are shown as solid lines for different $K$ band absolute magnitudes ranging from $K_{\mathrm{ABS}}=-25$ to -21 . While an optically selected sample with $I \leq 24$, can detect ellipticals brighter than $K_{\mathrm{ABS}} \geq-23.0$ up to $z \sim 1.2$, a $3.6 \mu$ m selected sample with $m(3.6 \mu \mathrm{m}) \leq 21.5$ can detect them up to $z \sim 2$ if very deep optical photometry is available. The present sample is therefore well adapted to investigate the evolution of old and/or massive galaxies up to $z \sim 2$, providing a major step with respect to previous analyses based on optical selection.

\section{Photometric redshifts}

\subsection{The method}

We measure the photometric redshifts and we classify the whole population in galaxy/quasar/star based on the $\chi^{2}$ fitting analysis of the spectral energy distributions, using the photometric redshift code "Le Phare"1. In this work, we adopt a similar procedure to the one described by Ilbert et al. (2006b). We use empirical templates based on the four observed spectral types from Coleman et al. (1980) and add two starburst templates from Kinney et al. (1996). Templates are extrapolated into ultraviolet and infra-red wavelengths using the GISSEL synthetic models (Bruzual \& Charlot 2003) and we refine the set of SEDs with a linear interpolaton amongst the original SEDs. For Scd and later spectral types, we allow for different amounts of dust attenuation with a reddening excess $E(B-V)$ varying from 0 to 0.6 and an interstellar extinction law from Prevot et al. (2004). The spectroscopic sample is used to perform a template optimization and estimate possible systematic shifts amongst the different passbands as introduced by Ilbert et al. (2006b). The SED fitting is performed from the $u^{*}$ to the 3.6 and $4.5 \mu \mathrm{m}$ photometric passbands (the two latter passbands being still dominated by stellar light). All objects have at least 4 passbands with measured fluxes which ensures reliable SED fitting analysis.

In Fig. 3, we compare the photometric and spectroscopic redshifts for 1400 galaxies observed by the VVDS. We obtain an

\footnotetext{
${ }^{1}$ http://www. oamp.fr/people/arnouts/LE_PHARE.html
}

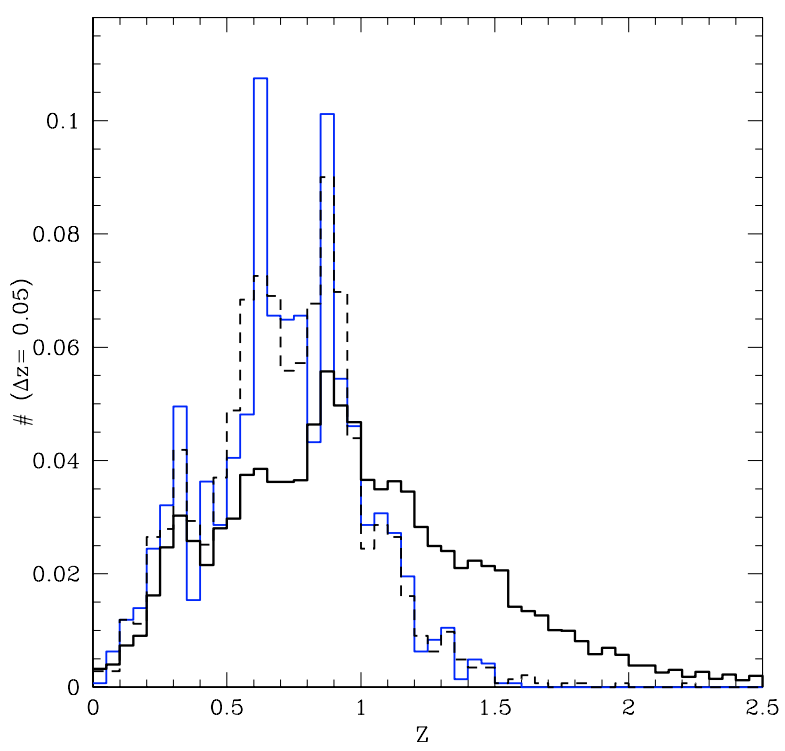

Fig. 4. The redshift distributions for the $3.6 \mu \mathrm{m}$ sample with $F(3.6 \mu \mathrm{m}) \geq 9 \mu \mathrm{Jy}$, for the full photo- $z$ sample (solid thick line), the spectroscopic sub-sample (solid thin line) and the photo- $z$ of spectroscopic objects (dashed line). All three curves are normalized to unity.

accuracy in the photo- $z$ of $\sigma[\Delta z /(1+z)] \sim 0.031$ with no systematic shift and a small fraction of catastrophic errors $(1.5 \%$ with $|\Delta z| \geq 0.15(1+z)$ ).

We consider as stars and QSOs (or AGN) the objects with a high SExtractor stellarity index parameter in $i^{\prime}$ band (CLASS_STAR $\geq 0.97$ ) and for which either the star or the QSO template provide a best $\chi^{2}$ fitting. After removing these stars and QSOs, we end up with a total sample of $\sim 21200$ galaxies out of 25500 sources.

\subsection{The redshift distributions}

The redshift distributions are shown in Fig. 4 for the whole sample with photometric redshifts (thick line), for the spectroscopic sub-sample ( $I \leq 24$, thin line) and for the photo- $z$ 's of the spectroscopic objects (dashed line). The two latter distributions, both optically selected, are in excellent agreement, but do not show the high redshift tail (up to $z=2-2.5$ ) observed for the $3.6 \mu \mathrm{m}$ sample. We find that $\sim 40 \%$ of galaxies with $F(3.6 \mu \mathrm{m}) \geq 9 \mu \mathrm{Jy}$ are at $z \geq 1$. A similar fraction, $\sim 35 \%$, is obtained by Franceschini et al. (2006) for galaxies with $F(3.6 \mu \mathrm{m}) \geq 10 \mu \mathrm{Jy}$ in the GOODS-CDFS field. Note however that both samples rely partially on photometric redshifts. Since the high- $z$ tail $(z \geq 1.4)$ cannot be verified by our spectroscopic sample, we have investigated the reliability of the redshifts for this high- $z$ population through color-color diagnostics. In Fig. 5, we show the $\left(g^{\prime}-z^{\prime}\right)$ vs. $\left(z^{\prime}-36 \mu \mathrm{m}\right)$ color-color diagram as an indicator for galaxies at $z \geq 1.4$. This is similar to the BzK diagnostic proposed by Daddi et al. (2004). The locus of galaxies with $z_{\text {phot }} \geq 1.4$ is well separated from the low redshift population as illustrated by the VVDS spectroscopic sample. This was also reported by Daddi et al. (2004) for the spectroscopic followup of BzK candidates. We conclude that our MIR selection at $3.6 \mu \mathrm{m}$ allows us to probe a significant population of galaxies in the redshift range $1 \leq z \leq 2$ with no indication of any bias from the lack of spectroscopic redshifts.

The spectroscopic redshift distribution also shows strong peaks at $z \sim 0.35,0.6$ and 0.85 . Two of them $(z=0.35$ and 0.85 ) are still observed in the photo- $z$ distribution although 


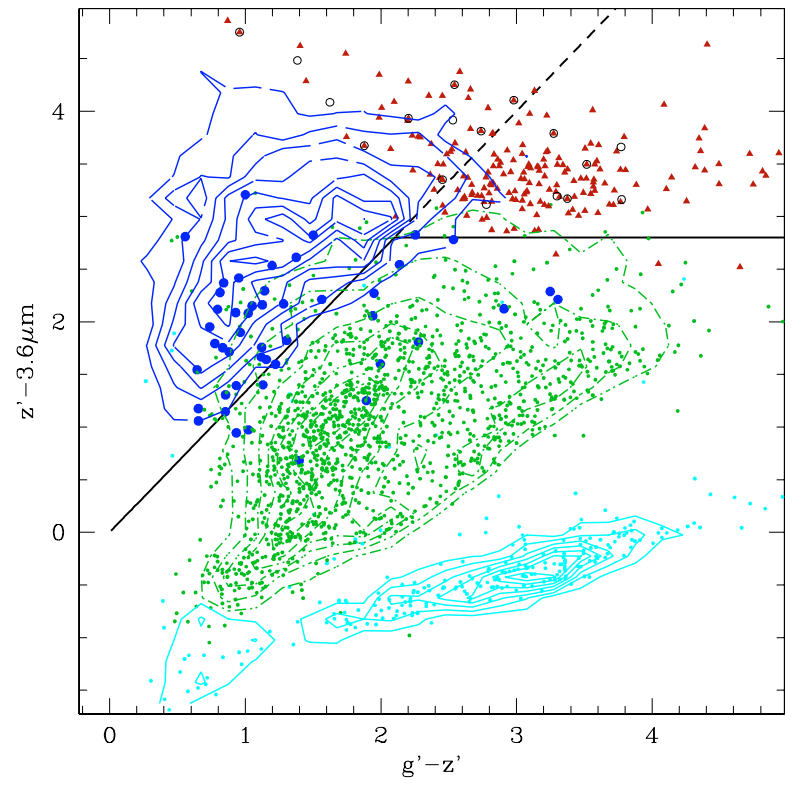

Fig. 5. Color-color diagram with $\left(z^{\prime}-3.6 \mu \mathrm{m}\right)$ vs. $\left(g^{\prime}-z^{\prime}\right)$ for $3.6 \mu$ sample. The filled dots show the spectroscopic objects while contours show the distributions of the photo- $z$ sample for $z \leq 1.4$ (dashed-dotted green contours), $z \geq 1.4$ for the active/blue (long dashed blue contours) and stars (thin cyan contours). The quiescent/red populations with $z_{\text {phot }} \geq 1.4$ are shown as triangles and the open circles show dusty galaxies observed at $24 \mu \mathrm{m}(F(24 \mu \mathrm{m}) \geq 300 \mu \mathrm{Jy})$ and falling above the $\left(N U V-r^{\prime}\right)_{\mathrm{ABS}}$ criteria described in the text. The lines show a typical separation between the three classes in a similar way as the BzK selection.

the area is two times larger and the distribution is somewhat smeared by the photo- $z$ errors. These two peaks have been identified as large scale structures extending through the entire field (Marinoni et al. 2005; Adami et al. 2007) and we estimate the number density to be in excess by $\sim 20-25 \%$ in these structures (based on a smoothed redshift distribution derived by applying a moving window with a width of $\Delta z=0.2$ ). Despite the large field of view, cosmic variance remains a significant source of uncertainty as previously pointed out by Bell et al. (2004) in the case of the COMBO17 survey. Following the recipes described by Somerville et al. (2004), we estimate the cosmic variance from large scale structure fluctuations in the $\Lambda \mathrm{CDM}$ paradigm. In the low and high redshift bins (with volumes of 2.7 and $12.5 \times 10^{5} \mathrm{Mpc}^{3}$ respectively), we expect the cosmic variance to produce density variations of $30 \%$ and $10 \%$ respectively, which appears to be in good agreement with our own estimates. We decide to account for this effect as an additional source of uncertainties in the measurements presented hereafter.

\subsection{Estimates of absolute magnitudes}

Throughout this paper we use absolute luminosity quantities. In practice, to derive the absolute magnitudes we use an adaptive filter method as introduced and tested on simulations by Ilbert et al. (2005, Appendix A). To reduce the uncertainties in the $k$-corrections used to derive the absolute magnitude at $\lambda_{\text {rest }}$, we choose the apparent magnitude in the filter $\left(\lambda_{\text {obs }}\right)$ that best matches the redshifted $\lambda_{\text {rest }}$. In the case where $\lambda_{\text {obs }}=\lambda_{\text {rest }} *(1+z)$, the $k$-correction is reduced to its redshift component $(2.5 * \log (1+$ z)) with no template dependency.

All absolute magnitudes are derived according to this scheme except for the specific case of the $K$ absolute magnitude

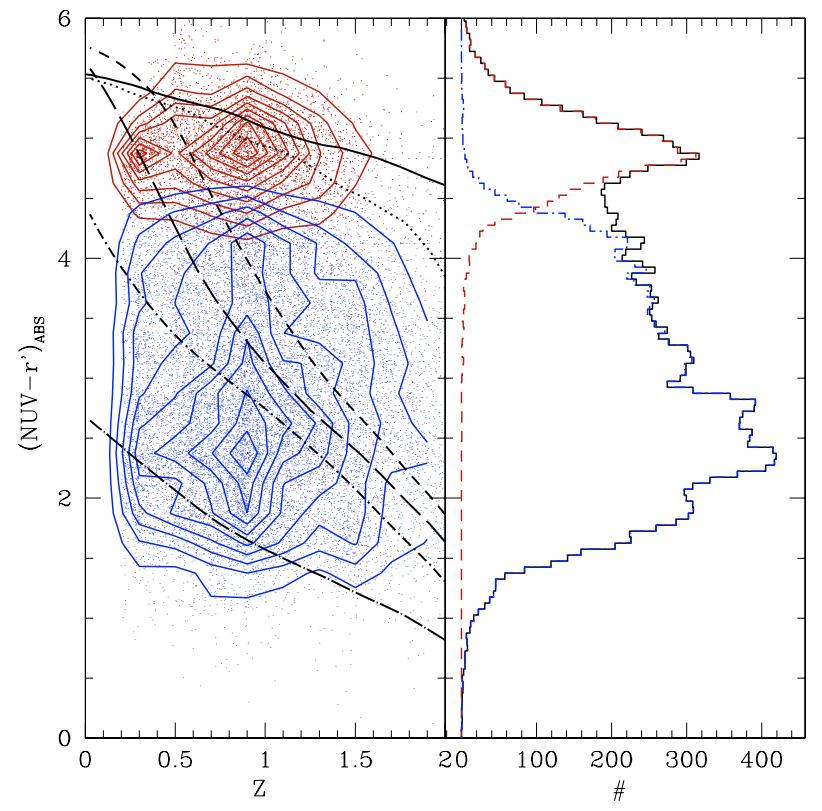

Fig. 6. Left panel: $\left(N U V-r^{\prime}\right)_{\mathrm{ABS}}$ color vs. redshift for the quiescent/red (red contours) and active/blue (blue contours) populations. Color evolution for models with $z_{\mathrm{f}}=3,6$ and $\tau=0.1$ (dotted and solid lines resp.); $z_{\mathrm{f}}=3$ and $\tau=1,1.5$ (short and long dashed lines); $\tau=2, \infty$ (dot-short dashed and dot-long dashed lines resp). Right panel: histogram for the $\left(N U V-r^{\prime}\right)_{\mathrm{ABS}}$ color (solid line). The dashed lines show the distributions of the two populations as separated by SED templates (see text).

$\left(K_{\mathrm{ABS}}\right)$. We always use use the $3.6 \mu \mathrm{m}$ magnitudes for $z \geq 0.4$. This choice yields null $k$-correction at $z \sim 0.6$ and requires small $k$-correction in highest redshifts with little template dependency. When available, at $z \leq 0.4$, we use the observed $K$ band.

In the next section we use the $N U V$ and $r^{\prime}$ absolute luminosities. Over the redshift range $0.3 \leq z \leq 1.75$ the rest-frame $N U V$ ( $2350 \AA)$ and $r^{\prime}$ passbands correspond to observed wavelengths of $0.3 \mu \mathrm{m} \leq \lambda \leq 0.65 \mu \mathrm{m}$ and $0.8 \mu \mathrm{m} \leq \lambda \leq 1.7 \mu \mathrm{m}$ respectively. We therefore need only small extrapolations for the $N U V$ absolute magnitudes in the lowest $z$-bin. For the $r^{\prime}$ filter, the near infrared photometry is required at high redshift, which is available for a large fraction of our sample. We note however, that even without near-infrared photometry the shape of the SED is always well constrained in the NIR domain, because all our objects have per definition a measurement at $3.6 \mu \mathrm{m}$. We therefore obtain a good estimate of the $r^{\prime}$ absolute magnitudes even at high redshift.

We have derived the absolute magnitudes based on the empirical SEDs used to measure the photo- $z$. As a external check, we have also measured the absolute magnitudes based on a library of SEDs from PEGASE2 code (Fioc et al. 1997). Thanks to the use of the adaptive filter method, the results from the two libraries are fully consistent with an rms dispersion for the $\left(N U V-r^{\prime}\right)_{\mathrm{ABS}}$ color of $\sigma\left[\Delta\left(N U V-r^{\prime}\right)\right] \sim 0.2$. This is small compared to the large dynamical range of this color (see Fig. 6).

\subsection{The selection of quiescent galaxies}

We split our sample in two main classes in order to distinguish the red/quiescent and blue/active galaxies. To do so, we explore and compare two approaches, where one is based on the color bimodality and the other on an SED fitting procedure. For the color bimodality we consider the excellent separation provided by the $\left(N U V-r^{\prime}\right)_{\mathrm{ABS}}$ color (Fig. 6; right panel) as suggested 


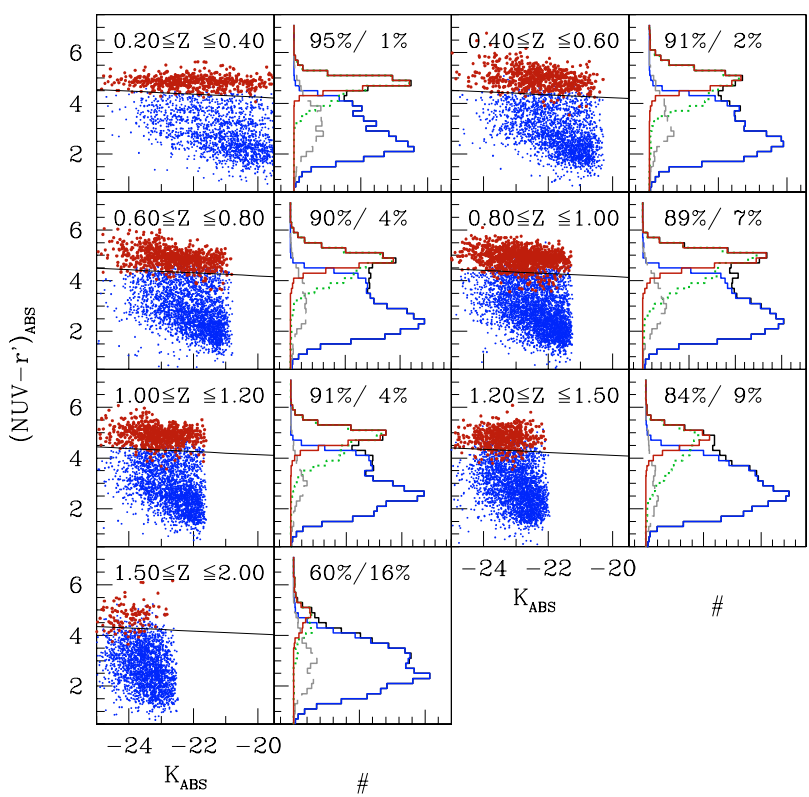

Fig. 7. $\left(N U V-r^{\prime}\right)_{\mathrm{ABS}}$ color vs. $K_{\mathrm{ABS}}$ distribution (left side) as a function of redshift (as specified in each panel). The two colored set of points correspond to the quiescent and active galaxies as separated by SED templates. The solid lines denote the valley between the two populations as seen in the projected histograms (right side). The green dotted histograms show the selection of red galaxies based on $U-B$ rest-frame color (see text). The dashed histograms show the galaxies detected at $24 \mu \mathrm{m}(F(24 \mu \mathrm{m}) \geq 300 \mu \mathrm{Jy})$.

by recent GALEX results (Salim et al. 2005; Wyder et al. 2007). This is in contrast to the often used rest-frame $(U-V)_{\mathrm{ABS}}$ color suggested by Bell et al. (2004).

One of the advantages of the $\left(N U V-r^{\prime}\right)_{\mathrm{ABS}}$ color is that the $N U V$ passband is sensitive to a stellar population with a lightweighted age of $\langle t\rangle \sim 10^{8} \mathrm{yr}$ while the $r^{\prime}$ passband is probing $\langle t\rangle \geq 10^{9} \mathrm{yr}$ (Martin et al. 2005), making the $\left(N U V-r^{\prime}\right)_{\mathrm{ABS}}$ color an excellent indicator of the current over past star formation activity. Indeed, the GALEX-SDSS sample shows that $\left(N U V-r^{\prime}\right)_{\mathrm{ABS}}$ is tightly correlated with the birthrate b parameter $\left(b=S F R\left(t<10^{8} \mathrm{yr}\right) /\langle S F R\rangle\right)$ with $\left(N U V-r^{\prime}\right)_{\mathrm{ABS}} \geq 4.3$ being associated to galaxies with $b \leq 0.1$ and morphologically with de Vaucouleur profiles (Salim et al. 2005).

To illustrate the redshift evolution in $\left(N U V-r^{\prime}\right)_{\mathrm{ABS}}$, we use the stellar synthesis model PEGASE2, with smooth star formation histories, described by different e-folding times and formation redshifts $\left(\tau(\mathrm{Gyr}), z_{\mathrm{f}}\right)$. In Fig. 6, left panel, we show the evolution of $\left(N U V-r^{\prime}\right)_{\mathrm{ABS}}$ vs. $\mathrm{z}$ for different SF histories $(\tau)$ and redshift of formation. Galaxies with $\tau \leq 0.1 \mathrm{Gyr}$ are in the red clump at all $\mathrm{z}$, while models with higher $\tau$ move progressively into the red sequence at decreasing redshifts $(z \sim 0.7$ and 0.4 for $\tau=1$ and 1.5 Gyr respectively). All models with $\tau \geq 2 \mathrm{Gyr}$ have ongoing star formation that prevents them from reaching the red sequence unless their star formation is quenched prematurely. Therefore the $\left(N U V-r^{\prime}\right)_{\mathrm{ABS}}$ color bimodality (Fig. 6, right panel) appears to be a good way to separate active and quiescent galaxies according to their birthrate parameter, with $b \leq 0.1$ for quiescent galaxies.

In Fig. 7, we show the galaxy distribution in the diagram $\left(N U V-r^{\prime}\right)_{\mathrm{ABS}}$ vs. $K_{\mathrm{ABS}}$ and the projected histograms for the different redshift bins. A strong bimodality is observed at almost all redshifts providing a natural way to split the sample. We define an adaptive color cut-off located in the valley and characterized by: $\left(N U V-r^{\prime}\right)_{\mathrm{ABS}}=-0.06 \times\left(K_{\mathrm{ABS}}+22\right)+b(z)$, where

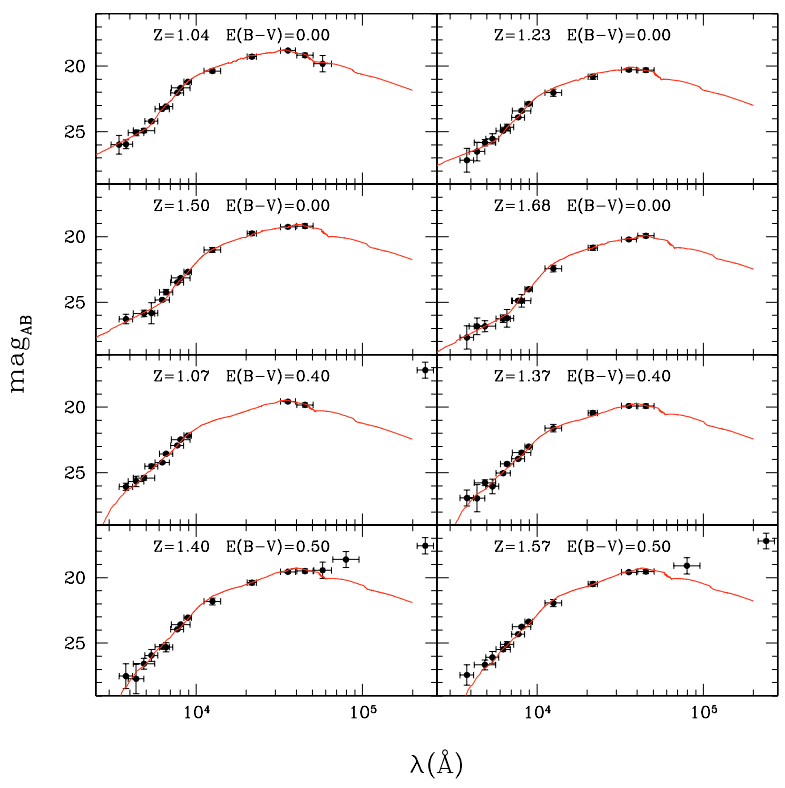

Fig. 8. Examples of SEDs for a sample of high- $z$ galaxies with (NUV $\left.r^{\prime}\right)_{\mathrm{ABS}}$ above of the color criterion (see text). The fit is performed only in the wavelength domain $0.3 \mu \mathrm{m} \leq \lambda \leq 4.5 \mu \mathrm{m}$. The top four objects are best fitted by an old/passive elliptical, while the lower four galaxies are best fitted by dusty star-forming SEDs.

$b(z)=4.4-0.13 \times z$ (solid lines). However, some uncertainties remain on the nature of the population in the red sequence due to the contamination by dusty star-forming galaxies. A reddening excess of $E(B-V)=0.2$ produces a color reddening of $\Delta(N U V-r)_{\mathrm{ABS}}=0.94$ (or 1.04), assuming starburst (or SMC) extinction curve. We also show in Fig. 7, the locations of those galaxies that are most likely dusty star-forming galaxies, as indicated by their detection at $24 \mu \mathrm{m}$ (with $F(24 \mu \mathrm{m}) \geq 300 \mu \mathrm{Jy}$ ). Between 5 to $9 \%$ (depending on $z$ ) of this bright $24 \mu \mathrm{m}$ population lies above our adopted colour cut and is a source of contamination for our quiescent population.

We then take a different approach, by classifying the galaxies according to the best-fit SED, on the basis of the six original templates (but allowing for dust extinction for the later types), in a similar way as Zucca et al. (2006). We consider as quiescent galaxies only those classified as elliptical and all other galaxies as active.

In Fig. 7, we compare the SED-based classification with the $\left(N U V-r^{\prime}\right)_{\mathrm{ABS}}$ color distribution (quiescent as red points; active as blue points). To quantify the overlap between the two different classifications, in each panel, we measure the fraction of ellipticals in the sample above and below the color cut previously defined. We find that the SED-ellipticals dominate (with 90\%) the red peak population up to $z \leq 1.5$ with only a small fraction falling below the cut. At $z \geq 1.5$, the fraction of ellipticals above the cut represents only $60 \%$ of the reddest objects while the remaining $40 \%$ are best classified as dusty star-forming galaxies. In Fig. 8, we show examples of the two types of SEDs (Ell or dusty star-forming) for galaxies above the $\left(N U V-r^{\prime}\right)$ cut and with $z_{\text {phot }} \geq 1.0$.

In Fig. 5, we show the distribution of SED-elliptical galaxies with $z \geq 1.4$ (red triangles). As for the BzK selection with a spectroscopic validation (Daddi et al. 2004), they are reasonably well separated from the active galaxies with $z \geq 1.4$ (blue contours). We also show the bright $24 \mu \mathrm{m}$ population with $\left(N U V-r^{\prime}\right)_{\mathrm{ABS}}$ in the red sequence and $z \geq 1.4$ (open circles). They are spread all over the upper part of the diagram. Amongst this population, 
$\sim 50 \%$ of them has been correctly adjusted by dusty star-forming SEDs. This shows that SED fitting can get rid partially of the dusty galaxies falling in the red sequence. As a last test to quantify the residual contamination of the red/quiescent sample, we project our elliptical sample in the $(J-K)$ vs. $\left(r^{\prime}-K\right)$ color diagram. This is susceptible to distinguish the early-types from the dusty starbursts (Mannuzzi \& Pozzetti 2000; Daddi et al. 2004). We find that $\sim 15 \%$ of the sample lies in the dusty starburst locus. If this color separation were to be efficient, this would mean that our quiescent sample may still be moderately contaminated by dusty galaxies.

In conclusion, we have analyzed two methods to select the $\mathrm{red} /$ quiescent galaxies based either on the $\left(N U V-r^{\prime}\right)_{\mathrm{ABS}}$ color or on the selection of quiescent galaxies according to SED fitting. We have shown that the SED-quiescent population represents the large majority of galaxies in the red peak of the bimodality distribution. We decide to adopt the SED-fitting classification because it allows to reduce the contamination of dusty starbursts in our red/quiescent sample. At $z \geq 1.4$, we estimate the residual contamination to be around $\sim 15 \%$. Only deeper Spitzer-MIPS observations could allow to better disentangle the two populations. However we note that the results discussed in the following sections are marginally affected by the chosen selection.

We end up with a sample of 4425 quiescent and 16770 active galaxies.

\section{The stellar mass to light ratio $\left(M / L_{K}\right)$}

We now derive the evolution of the stellar mass to light ratio $\left(M / L_{K}\right)$ with redshift for the active and quiescent populations. This will allow us to assess stellar mass quantities. Thanks to the large VVDS spectroscopic sample, we can measure how the $M / L_{K}$ varies with redshift. In particular, the use of spectroscopic redshifts allows us to avoid mass uncertainties relative to photo- $z$ and we thus obtain accurate estimates of the scatter for these relations.

We derive the stellar mass $M^{\star}$ for each galaxy by fitting the multi-band photometry. In the present work, we base ourselves on the Bruzual \& Charlot (2003) stellar population model. We have used this model to construct a library of star formation histories that includes stochastic bursts (see Kauffmann et al. 2003; and Salim et al. 2005, for similar libraries). Although this library uses a Chabrier (2003) IMF, we have decided to adopt a classical Salpeter IMF throughout this paper. We therefore apply a systematic shift of +0.24 dex to the masses derived with a Chabrier IMF. In brief, our method uses the Bayesian probability distribution functions as described in Appendix A of Kauffmann et al. (2003) to derive parameter estimates for each observed SED, including errors on each derived parameters, in this case the stellar mass. A full description of our mass estimation routines will appear in forthcoming papers (Lamareille et al. 2007; Walcher et al. 2007). For now we refer to previous works for discussion on the accuracy of the stellar mass estimates by SED fitting methods, in particular the choice of star formation histories with or without episodic bursts (Borch et al. 2006; Pozzetti et al. 2007) and the addition of Mid-IR bands (Fontana et al. 2006). We note, however, that our stellar mass estimates may be affected by the established problems pertaining to the contribution of short-lived, luminous, infrared-bright stars in intermediate age population ( $\sim 1$ Gyr) (Maraston et al. 2006). Our mass estimates would possibly need to be changed by 0.1 to $0.2 \mathrm{dex}$ (see Pozzetti et al. 2007 for an in-depth discussion). A final solution to this problem affecting all publicly available stellar population synthesis models is however not imminent.

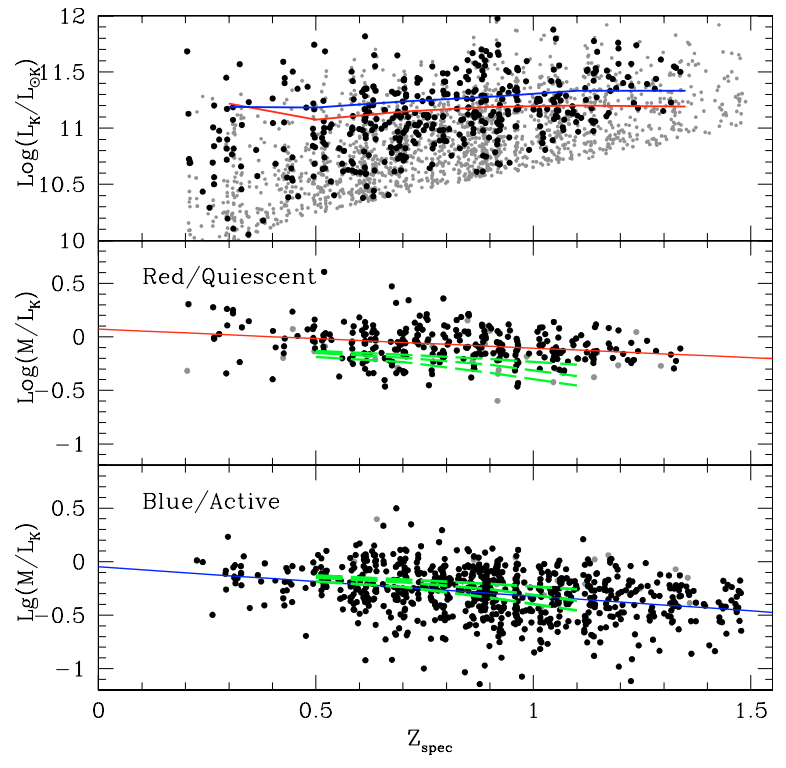

Fig. 9. Upper panel: distribution of the $K$ luminosity $\left(L_{K} / L_{\odot K}\right)$ for the spectroscopic sample (quiescent galaxies: black filled circles; active galaxies: grey small circles). The characteristic luminosity $L^{\star}$ for the Red/quiescent and Active/blue samples are shown as solid lines. Lower panels: behavior of the Mass to light ratio $\left(M / L_{K}\right)$ for the quiescent (middle panel) and active (lower panel) samples with the best fit shown as solid lines. Comparison with results from Drory et al. (2004; long dashed lines) based on three different stellar mass cuts: $M / M_{\odot} \geq$ $2 \times 10^{11}, 10^{11} \leq M / M_{\odot} \leq 2 \times 10^{11}$, and $4 \times 10^{10} \leq M / M_{\odot} \leq 10^{11}$.

In Fig. 9 (upper panel), we show the redshift distribution of the K luminosity $\left(L_{K} / L_{\odot K}\right)$ for the quiescent (large black circles) and active (small grey circles) populations. The solid lines show the evolution of the characteristic luminosity, $L^{\star}$, derived below in Sect. 5.1 and reported in Table 1. The distribution of $M / L_{K}$ as a function of redshift for the quiescent and active samples are shown in the middle and lower panels respectively. Before deriving the $M / L_{K}$ versus redshift relations from the spectroscopic sample we have tested several subsets: the first one includes only objects brighter than $L_{K} \geq 10^{11} L_{\odot K}$ to define an unbiased luminosity sample up to $z=1.5$. The second one considers objects with a luminosity between $0.4 L^{\star}(z) \leq L_{K}(z) \leq 2.5 L^{\star}(z)$, allowing to probe the evolution of the $M / L_{K}$ for galaxies around $L^{\star}(z)$ which are the main contributors to the total luminosity density with $70 \%, 45 \%$ and $55 \%$ for the quiescent, active and total samples, respectively. Because the spectroscopic sample is optically selected $(I \leq 24)$, we have also considered a sub-sample with $m 3.6 \leq 20.5$ (which means one magnitude brighter than the full sample) to insure a fair sampling of the $\left(i^{\prime}-3.6 \mu \mathrm{m}\right)$ colors at all redshifts. All these subsamples are found to provide consistent results within the parameter's uncertainties, therefore we only report the values for the three samples with the selection centered around their respective $L^{\star}(z)$. For the three samples, we fit the redshift evolution of the $M / L$ ratio by a power-law defined as $\log \left\langle M / L_{K}\right\rangle=a \times Z+b$, with a global rms given by $\sigma$.

For the global sample, based on 999 galaxies:

$a=-0.30 \pm 0.03, b=0.03 \pm 0.03, \sigma=0.22$.

For the active/blue sample, based on 753 galaxies:

$a=-0.27 \pm 0.03, b=-0.05 \pm 0.03, \sigma=0.21$.

For the quiescent/red sample, based on 298 galaxies:

$a=-0.18 \pm 0.04, b=+0.07 \pm 0.04, \sigma=0.15$. 
Table 1. K-LF parameters $\left(\alpha, M^{\star}, \Phi^{\star}\right)$ and $K$ luminosity densities $\left(\rho_{L}\right)$ for the whole, active and quiescent samples. Errors in $M^{\star}$, $\Phi^{\star}$ refer to Poisson errors and slope uncertainties. Additional uncertainties due to photometric redshift $(P \mathrm{~d} Z)$ and cosmic variance $(\mathrm{CV})$ are reported separately. Errors in $\rho_{L}$ include all of them.

\begin{tabular}{|c|c|c|c|c|c|c|c|}
\hline$\langle z\rangle$ & \# & $\alpha$ & $M^{\star}$ & $\begin{array}{c}\Phi^{\star} \\
\left(10^{-3} \mathrm{Mpc}^{-3}\right)\end{array}$ & $\left(\frac{\mathrm{d} \Phi}{\Phi}\right)_{P \mathrm{~d} Z}$ & $\left(\frac{\mathrm{d} \Phi}{\Phi}\right)_{\mathrm{CV}}$ & $\begin{array}{c}\rho_{L} \\
\left(10^{8} L_{\odot K} \mathrm{Mpc}^{-3}\right)\end{array}$ \\
\hline $\begin{array}{c}0.3 \\
0.5 \\
0.7 \\
0.9 \\
1.1 \\
1.35 \\
1.75\end{array}$ & $\begin{array}{l}2180 \\
2680 \\
3336 \\
4545 \\
3027 \\
3077 \\
2189 \\
\end{array}$ & $\begin{array}{l}-1.1 \pm 0.2 \\
-1.1 \pm 0.2 \\
-1.1 \pm 0.2 \\
-1.1 \pm 0.2 \\
-1.1 \pm 0.2 \\
-1.1 \pm 0.2 \\
-1.1 \pm 0.2\end{array}$ & $\begin{array}{c}\text { ALL } \\
-22.84_{-0.6}^{+0.4} \\
-22.83 \pm 0.3 \\
-22.96 \pm 0.2 \\
-23.08 \pm 0.2 \\
-23.22 \pm 0.2 \\
-23.18 \pm 0.17 \\
-23.42 \pm 0.14\end{array}$ & $\begin{array}{c}4.19_{-1.9}^{+2.2} \\
3.50 \pm 1.3 \\
3.36 \pm 1.1 \\
4.22 \pm 1.2 \\
2.75 \pm 0.7 \\
2.56 \pm 0.5 \\
1.47 \pm 0.2\end{array}$ & $\begin{array}{l}0.05 \\
0.05 \\
0.05 \\
0.05 \\
0.11 \\
0.09 \\
0.07\end{array}$ & $\begin{array}{l}0.23 \\
0.20 \\
0.16 \\
0.26 \\
0.13 \\
0.12 \\
0.10\end{array}$ & $\begin{array}{l}6.97 \pm 2.2 \\
5.77 \pm 1.3 \\
6.24 \pm 1.3 \\
8.75 \pm 2.5 \\
6.48 \pm 1.4 \\
5.82 \pm 1.5 \\
4.17 \pm 1.1\end{array}$ \\
\hline $\begin{array}{c}0.3 \\
0.5 \\
0.7 \\
0.9 \\
1.1 \\
1.35 \\
1.75 \\
\end{array}$ & $\begin{array}{l}1666 \\
2063 \\
2569 \\
3428 \\
2358 \\
2609 \\
2081 \\
\end{array}$ & $\begin{array}{l}-1.3 \pm 0.2 \\
-1.3 \pm 0.2 \\
-1.3 \pm 0.2 \\
-1.3 \pm 0.2 \\
-1.3 \pm 0.2 \\
-1.3 \pm 0.2 \\
-1.3 \pm 0.2\end{array}$ & $\begin{array}{c}\text { active } \\
-22.83_{-0.56}^{+0.43} \\
-22.82 \pm 0.3 \\
-22.95 \pm 0.2 \\
-23.06 \pm 0.2 \\
-23.19 \pm 0.2 \\
-23.19 \pm 0.18 \\
-23.49 \pm 0.15\end{array}$ & $\begin{array}{c}2.38_{-1.3}^{+1.6} \\
2.22 \pm 1.0 \\
2.21 \pm 0.9 \\
2.82 \pm 1.0 \\
1.99 \pm 0.6 \\
2.08 \pm 0.5 \\
1.27 \pm 0.3 \\
\end{array}$ & $\begin{array}{l}0.05 \\
0.05 \\
0.05 \\
0.05 \\
0.12 \\
0.09 \\
0.07\end{array}$ & $\begin{array}{l}0.27 \\
0.21 \\
0.17 \\
0.28 \\
0.14 \\
0.13 \\
0.11 \\
\end{array}$ & $\begin{array}{l}4.76 \pm 1.7 \\
4.40 \pm 1.3 \\
4.94 \pm 1.3 \\
6.97 \pm 2.7 \\
5.55 \pm 1.8 \\
5.80 \pm 2.0 \\
4.66 \pm 1.8\end{array}$ \\
\hline $\begin{array}{c}0.3 \\
0.5 \\
0.7 \\
0.9 \\
1.1 \\
1.35 \\
1.75 \\
\end{array}$ & $\begin{array}{c}514 \\
617 \\
767 \\
1117 \\
669 \\
468 \\
108 \\
\end{array}$ & $\begin{array}{c}-0.6 \pm 0.2 \\
-0.3 \pm 0.2 \\
-0.3 \pm 0.2 \\
-0.3 \pm 0.2 \\
0.0 \pm 0.3 \\
0.3 \pm 0.3 \\
0.6 \pm 0.3 \\
\end{array}$ & $\begin{array}{c}\text { quiescent } \\
-22.91_{-0.49}^{+0.34} \\
-22.55 \pm 0.19 \\
-22.73 \pm 0.15 \\
-22.83 \pm 0.14 \\
-22.86 \pm 0.17 \\
-22.84 \pm 0.16 \\
-23.27 \pm 0.15 \\
\end{array}$ & $\begin{array}{c}1.78_{-0.5}^{+0.42} \\
1.47 \pm 0.17 \\
1.29 \pm 0.13 \\
1.58 \pm 0.14 \\
0.90 \pm 0.08 \\
0.41 \pm 0.03 \\
0.05 \pm 0.02\end{array}$ & $\begin{array}{l}0.09 \\
0.07 \\
0.09 \\
0.07 \\
0.12 \\
0.21 \\
0.28 \\
\end{array}$ & $\begin{array}{l}0.33 \\
0.24 \\
0.21 \\
0.34 \\
0.17 \\
0.18 \\
0.17 \\
\end{array}$ & $\begin{array}{c}2.63 \pm 1.1 \\
1.60 \pm 0.4 \\
1.65 \pm 0.4 \\
2.22 \pm 0.8 \\
1.42 \pm 0.3 \\
0.74 \pm 0.2 \\
0.17 \pm 0.05 \\
\end{array}$ \\
\hline
\end{tabular}

The fits to the quiescent and active samples are shown as solid lines in Fig. 9. All subsamples show a similar trend with a gradual decline of the $\left\langle M / L_{K}\right\rangle$ by a factor $\sim 1.5$ to 2 up to $z=1$. Similar results were obtained by Drory et al. (2004) for samples selected with different mass limits, based on the NIR MUNICS survey, and are shown in Fig. 9 (long dashed lines) The behavior of the $M / L$ is mainly driven by the stellar activity. The youngest and bluest stellar population have the smallest $M / L_{\lambda}$ (Bell \& de Jong 2001; Drory et al. 2004). Similarly, the decline of the $M / L_{\lambda}$ with redshift reflects the well established decrease of the specific star formation rate (downsizing effect; Cowie et al. 1996: for a galaxy with same mass, the SF activity is higher in the past). The smaller decline of $M / L_{K}$ with redshift observed for the quiescent sample reflects an older mean age of the stellar population, pushing the epoch of formation of the stellar component to high redshift. We find that the quiescent and active samples show a rather small scatter along this relation, with only 0.15 dex and 0.22 dex scatter respectively. The main origin for the scatter is the dependance of the $M / L_{K}$ on the star formation history ( $\mathrm{SFH}$ ). The more complex SFH for the active population could be responsible for the larger scatter.

\section{The $K$ band luminosity function and density}

\subsection{The $K$ rest-frame luminosity function}

\subsubsection{K-LF measurements}

We measure the K rest-frame luminosity function (LF) by adopting the $V_{\max }$ and STY estimators from the VVDS LF tool (ALF; Ilbert et al. 2004). In Fig. 10, we show the LFs for the whole and spectroscopic samples and in Fig. 11 for the red/quiescent and blue/active samples. To measure the LFs, a weight has been applied as a function of apparent magnitude to account for the incompleteness in number counts. In the case of the spectroscopic

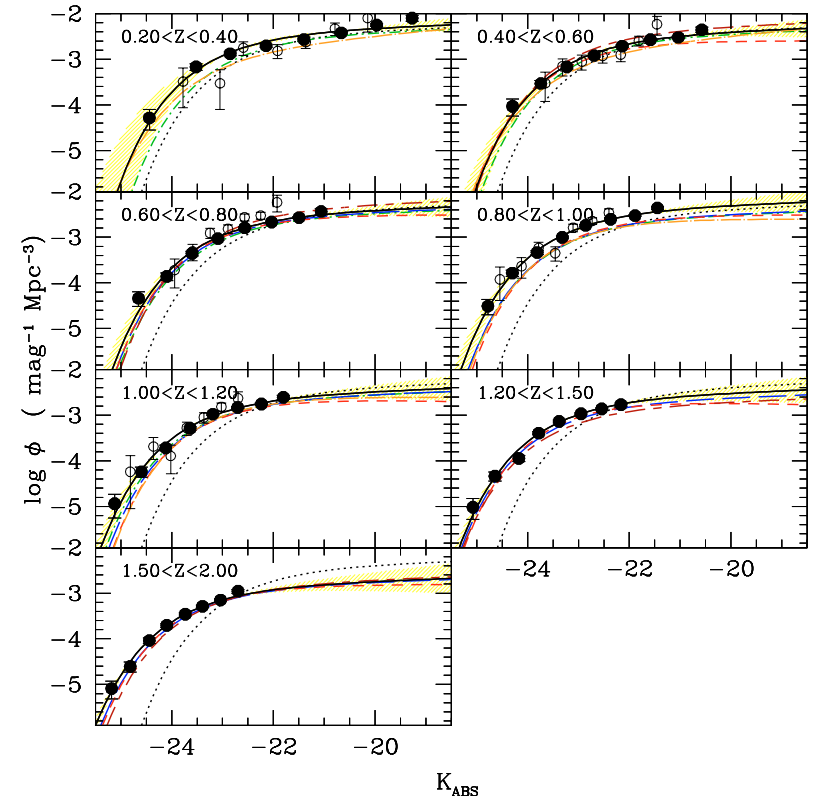

Fig. 10. $K$-band rest-frame luminosity functions, in different redshift slices, for the whole photo- $z$ sample $\left(V_{\max }\right.$ : filled dark circles, STY: solid lines and shaded area based on slope uncertainties) and the spectroscopic sample ( $V_{\max }$ : open circles). Also shown are the local LF from Kochanek et al. (2001; dotted line) and high- $z$ LFs from Drory et al. (2003; green dahed-dot line), Caputi et al. (2005; blue long dashed line), Pozzetti et al. (2004; orange long dashed-dot line), Saracco et al. (2006; dark red long-short dahed line, $\alpha=-1.1$ ), Cirasuolo et al. (2006; red dashed line).

sample, we derive the LFs only for galaxies with $m_{\mathrm{AB}}(3.6 \mu) \leq$ 20.5 and $0.2 \leq z \leq 1.2$. This restriction is due to the $I$ band selection effect (see Fig. 2). An additional weight is applied to the 


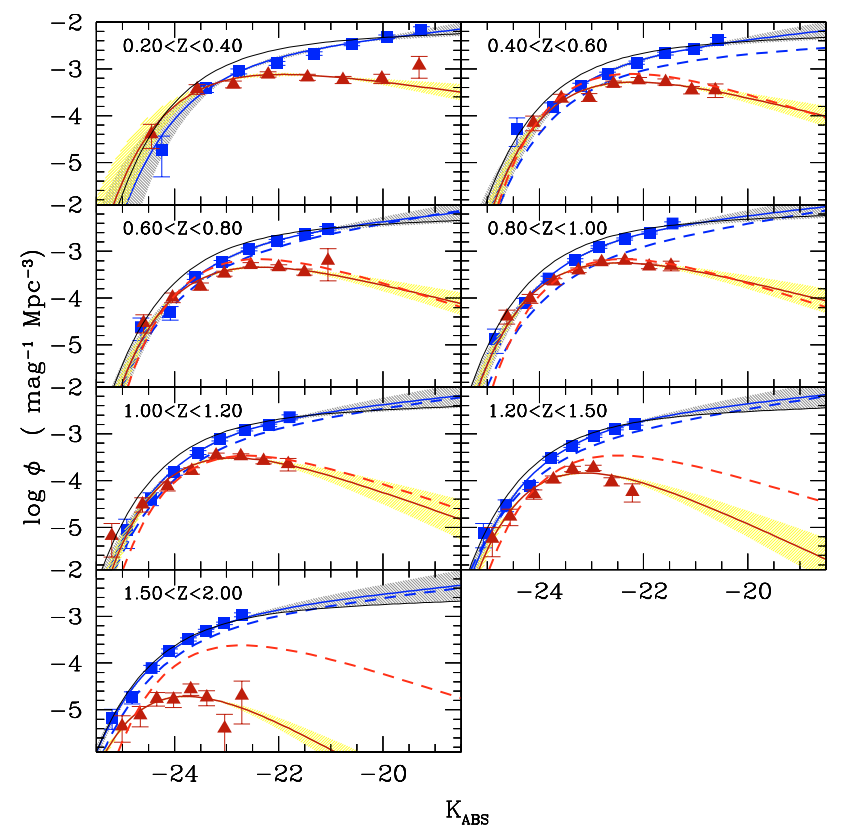

Fig. 11. K-rest luminosity functions for the blue/active (blue squares and solid lines) and the red/quiescent (red triangles and solid lines) samples. The thin black lines refer to the global sample from Fig. 10. We compare our LFs with the red and blue samples from the UKIDSS survey (Cirasuolo et al. 2006; red and blue dashed lines).

spectroscopic galaxies to take into account the sampling rate of the VVDS observations and its optical selection (as described in Ilbert et al. 2005).

In Table 1 we report the values of the STY parameters for the different samples. The errorbars on the parameters refer to the Poisson errors and slope uncertainties. For the global and blue samples, we have fixed the faint-end slopes of the STY estimator at low and high redshifts to the values observed between $0.6 \leq z \leq 1.0$, where we have the best constraints in the bright and faint-end simultaneously. This choice is consistent with local (Kochanek et al. 2001) and high redshift analysis (Drory et al. 2004; Caputi et al. 2005; Cirasuolo et al. 2006). For the red sample, we adopt a variable slope, although the low and high- $z$ slopes are less well constrained. At low $z(z \leq 0.4)$, we use $\alpha=-0.6$, consistent with the slope observed by Bell et al. (2004) from a local elliptical sample. At $z \geq 1$, we adopt a gradual flattening of the slope that best reflects the evolution of the $V_{\max }$ estimator. However the depth of the current data does not allow any statement on the reality of this apparent flattening and deeper observations are required to confirm it. We note that the choice of the slope does not affect the discussion in next sections because changes to the estimates of the luminosity densities are marginal $\left(\rho_{L}(\alpha)-\rho_{L}(\alpha=-0.3)=-0.01,-0.02,-0.04\right.$ dex for $\langle z\rangle=1.1,1.35,1.75$, resp.).

\subsubsection{Additional source of uncertainties}

Additional sources of uncertainties on the LFs come from the photo- $z$ and cosmic variance. We have estimated the uncertainties relative to the photo-zs from 100 mock samples based on the redshift probability distribution (PdZ) of each source (as in Bolzonella et al. 2000) and by recomputing the type separation and luminosity functions. Considering only the variations of $\Phi^{\star}$, we find uncertainties in the density to be between $5 \%$ to $12 \%$ for the total and active samples, depending on the redshift bin. For the quiescent sample, the uncertainties vary between $7 \%$ and $28 \%$. This method allows to account simultaneously for the large photo- $z$ uncertainties of the ellipticals at high- $z$ and to estimate the stability of the quiescent vs. active separation at high redshift. We report these uncertainties in Table $1\left((\mathrm{~d} \Phi / \Phi)_{P \mathrm{~d} Z}\right)$. Except for the quiescent sample at high $z$, the photo- $z$ uncertainties are not a dominant source of errors in the present work.

It is more difficult to evaluate the effect of cosmic variance. As a first attempt we have measured the global LFs in 10 non overlapping fields covering the whole area and find variations of $\Phi^{\star}$ of between 0.07 dex to 0.2 dex. However, the fact that the 10 fields are located in a single area of the sky prevents us from using them as cosmic variance uncertainties over the whole survey (adopting a simple rescaling as $\sqrt{10}$ ). Therefore, as mentioned in Sect. 2, we adopt the formal approach discussed by Somerville et al. (2004). The cosmic variance is estimated as $\sigma=b \sigma_{\mathrm{DM}}$, where $\sigma_{\mathrm{DM}}$ is the variance of the dark matter that depends on the comoving volume (their Fig. 3, right panel) and $\mathrm{b}$ is the bias relative to each specific sample that depends on the comoving number density (their Fig. 3, left panel). Theses uncertainties are reported in Table $1\left((\mathrm{~d} \Phi / \Phi)_{\mathrm{CV}}\right)$. Cosmic variance remains the dominant source of uncertainty for the whole and active samples at all redshift, while for the quiescent population the photo- $z$ uncertainty is the major source of uncertainty at $z \geq 1.35$.

\subsubsection{Evolution of the K-band LFs and comparison with other studies}

In Fig. 10, we compare the global LFs with other studies. Beyond the fluctuations of $\Phi^{\star}$ due to the presence of large scale structures, our LFs appear slightly brighter than previous studies, by about $10 \%$. This is however consistent with the expected calibration uncertainty of the SWIRE-IRAC flux (Surace et al. 2005). Comparing $K^{\star}$ to the local measurement by Kochanek et al. (2001), we observe a brightening of $\Delta K^{\star} \sim-0.8(-1.0) \pm$ 0.2 from $z=0$ to $z=1.5(2)$. Regarding the comoving density parameter $\left(\Phi^{\star}\right)$ we observe a decline by a factor $1.7 \pm 0.2$ up to $z=1.2$ and $2.5 \pm 0.5$ up to $z=2.0$ as compared to local estimate. This mild luminosity brightening and modest density decline agree with previous studies (Caputi et al. 2005; Saracco et al. 2006; Cirasuolo et al. 2006).

In Fig. 11, we compare the LF per types with our global LF and results from UKIDSS based on $(U-B)_{\text {rest }}$ color selection (Cirasuolo et al. 2006). Our active sample shows a similar brightening than the global sample. The density, $\Phi^{\star}$, appears stable between $0.2 \leq z \leq 1.5$ and starts to decline at higher $z$. For the quiescent sample a different behaviour is observed. Up to $z=1.2$, the density declines by a factor $2_{-0.7}^{+1}$, followed by a sharp drop, by a factor $16_{-6}^{+9}$, between $1.2 \leq z \leq 2$. While the behaviour of the active and quiescent samples agrees with UKIDSS results on blue and red galaxies (concerning faint end slopes and global evolution), we obtain a different ratio between the blue/active and red/quiescent populations and they do not observe the strong decline for the red population at $z \geq 1$.2. We suggest two differences in the selection criterion for red/quiescent galaxies as possible source to this discrepancy:

- In Fig. 7, we show the $\left(N U V-r^{\prime}\right)$ color distribution (green dotted histograms) for galaxies selected on the basis of the optical color $(U-B)$, in a similar way as Cirasuolo et al. While the Cirasuolo selection includes our quiescent sample, it also includes galaxies with $(N U V-r)$ as blue as 2.5-3. 


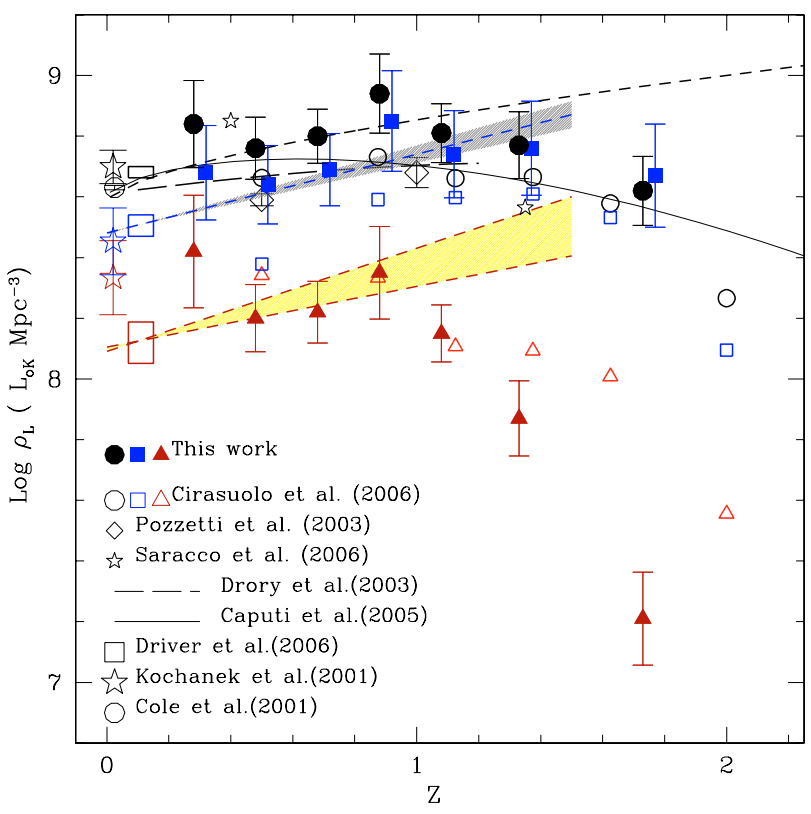

Fig. 12. Evolution of the luminosity density, $\rho_{L_{K}}$ (expressed in $L_{\odot K}$ ), for the total (filled circles), active (filled squares) and quiescent (filled triangles) populations. Results are compared with previous works as specified in the caption and with a pure luminosity evolution models (dashed lines).

This means that the optical criterion yields a number of red galaxies that is larger by $\sim 40$ to $50 \%$ depending on redshift;

- the modest decline of the red LF at $z \geq 1.2$ for UKIDSS can also takes its origin in the color criterion and in the inclusion of the larger fraction of red dusty starbursts at high- $z$. Regarding our selection of a quiescent sample, we were conservative in the sense that we required that the galaxies do not reveal signs of star formation activities according to their $\left(N U V-r^{\prime}\right)$ color and by using multi-color SED fitting to exclude dusty starbursts. In our red population at $1.5 \leq z \leq 2$, only $60 \%$ of galaxies is considered as quiescent, the rest being dusty starbursts. We expect this effect to be even stronger in the case of an optical color selection.

The strong evolution between $z=2$ and $z=1.2$ of the LF parameters of the quiescent sample alone suggests that we are probing the epoch when an increasing number of galaxies stop their star formation activities and turn quiescent. However, the LF parameters are strongly correlated with each others and their interpretation shaky. We therefore now turn to discussing the evolution of the different samples via the measurements of integrated quantities (luminosity densities and stellar mass densities).

\subsection{The $K$ band luminosity density}

A sensitive test for galaxy formation is the measurement of the luminosity density which provides the total amount of light emitted per unit of volume and is estimated as: $\rho_{L_{K}}=\int_{0}^{\infty} L \Phi(L) \mathrm{d} L=$ $\Phi^{\star} L_{K}^{\star} \Gamma(\alpha+2)$, where $\Gamma$ is the gamma function and $\left(\Phi^{\star}, L^{\star}, \alpha\right)$ are the Schechter parameters listed in Table 1. The measurements of $\rho_{L_{K}}$ (expressed in solar unit with $M_{K \odot}^{A B}=5.14$ ) are listed in Table 1 and plotted in Fig. 12. The quoted errorbars include all the sources of errors discussed in previous sections.
The total sample shows a luminosity density increase by a factor $\sim 2$ (starting from local values) up to $z \sim 1-1.2$ followed by a fall off of a similar amount up to $z=2$. This global behavior agrees, within the errorbars, with previous $K$ band luminosity density studies and is well described by the fit proposed by Caputi et al. (2005, solid line). Looking at the SED selected samples, the active sample dominates the $K$ band luminosity density at all $\mathrm{z}$ and follows a similar evolution as the global sample. For the quiescent sample, the luminosity density remains roughly flat up to $z \sim 1.2$ then drops significantly. As discussed in previous section, this trend is less pronounced in the UKIDSS's red sample measurements.

To interpret the evolution of the luminosity density for the different samples, we need to set local references. Since no similar $K$-band based selection exists, we make use of existing optical catalogs with information that can be converted to yield a $K$ band local reference. We have used the results reported by Driver et al. (2006) based on the Millennium Galaxy Catalog (MGC) where they provide the LFs in $B$ band and stellar mass density $\left(\rho_{\star}\right)$ for a large variety of classification, based on morphology, colors, SED fitting and spectral classes ( $\eta$ parameter from 2dF: Madgwick et al. 2002). We adopt two criteria for the local reference that can reflect the definition of our quiescent sample:

- as noted by Madgwick et al. (2003), the $\eta$ parameter is highly correlated with the birthrate $b$ parameter. In particular their first class with $\eta \leq-1.4$ corresponds to $b \leq 0.1$ and is thus similar to what we expect locally for our criterion. We adopt the $\eta_{1}$ class as representative of our quiescent sample and define all other classes as belonging to the active sample as reported in Table 2 of Driver et al. (2006);

- the majority of galaxies in the $\left(N U V-r^{\prime}\right)$ red sequence observed by GALEX are dominated by de Vaucouleur profiles (Salim et al. 2005). We adopt the E/SO(red) morphological class of the MGC as reported in their Table 2 as another representation of our quiescent sample.

Finally, we converted $\rho_{\star}$ to $\rho_{L_{K}}$ for each sample by adopting the Bell \& de Jong (2001) relations between $(B-R)$ and $M / L_{K}$. The resulting ranges of local estimates are shown in the Fig. 12 as colored rectangles. We additionally plot the morphologically separated luminosity densities from 2MASS (Kochanek et al. 2001) as colored open stars. The low- $z$ estimates agree reasonably well between themselves except for the quiescent sample from 2MASS. This is most likely due to the inclusion of the blue compact ellipticals in the sample of Kochanek et al. (2001). The MGC with its red E/S0 sample appears to be most suited to provide our local reference in this case.

Using the local normalization, we can now compare the evolution of $\rho_{L_{K}}$ for pure luminosity evolution (PLE) models, i.e. with no merging involved. We normalize to the local density $\Phi^{\star}(z=0)$ and use $\Delta \log \rho_{L_{K}}(z)=-0.4 \Delta K_{\mathrm{ABS}}(z)$. We adopt the luminosity evolution as derived in Sect. 5.1. Specifically, for the total sample we adopt the luminosity evolution from Caputi et al. (2005), for the active sample we adopt $\Delta K_{\mathrm{ABS}}(z)=$ $-0.65( \pm 0.07) \times Z$, while for the quiescent sample we use two extrem PEGASE models that encircles the observed luminosity evolution and can be described by $\Delta K_{\mathrm{ABS}}(z)=-0.5 \mid-0.85 \times Z$. In Fig. 12, the PLE predictions are shown with dashed lines and shaded areas. The evolution of the rest-frame $K$-band luminosity density is consistent with the PLE model for the total and active samples up to $z=1.2$, while the model overpredicts $\rho_{L_{K}}$ at higher redshift. This shows that the number density of galaxies has to drop. For the quiescent sample, the PLE model fails 


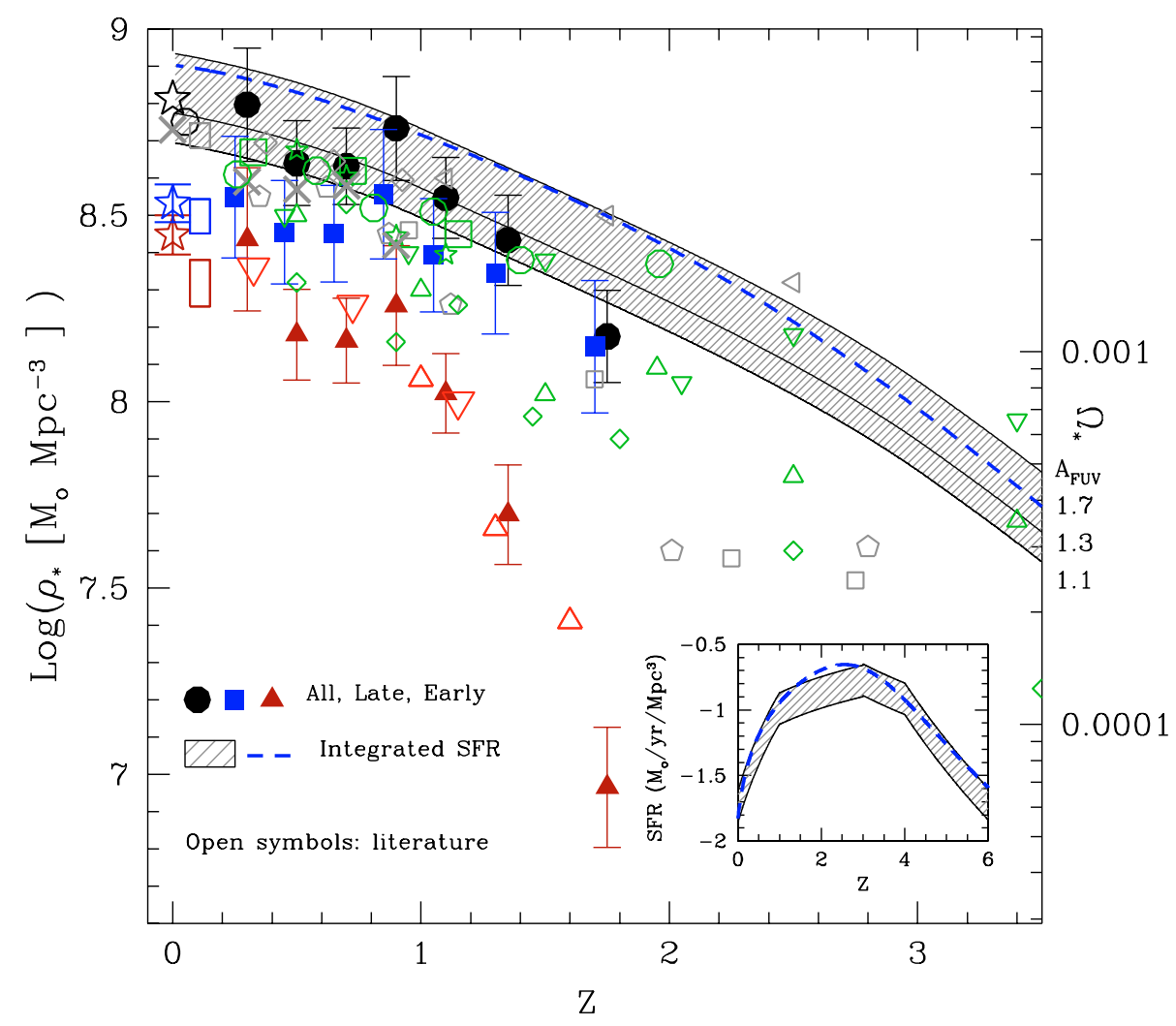

Fig. 13. Evolution of the stellar mass density as a function of cosmic time (assuming a Salpeter IMF). The total, active and quiescent stellar mass densities from this work are shown with large filled circles, blue squares and red triangles respectively. For reference, the right-hand axis gives the stellar density parameter. The integrated Star Formation Rates for different dust attenuation corrections $\left(A_{\mathrm{FUV}}=1.1,1.3,1.7\right)$, based on the SFR derived by GALEX (Schiminovich et al. 2005), are plotted as solid lines, and the one from the compilation of Hopkins and Beacom as a dashed line. The dust corrected SFRs are shown in the inset. High $z$ measurements from the literature for total samples have been splitted between analysis based on optical information only (grey symbols) and including Near or Mid IR data (green symbols) for the mass estimates. with Optical: Brinchmann \& Ellis (2000; pentagons); Cohen et al. (2002; losanges); Dickinson et al. (2003; squares); Gwyn et al. (2005; inclined triangles); Borch et al. (2006; crosses). with NIR data: Drory et al. (2004; open stars); Drory et al. (2005; up and down triangles); Fontana et al. (2006; losanges); Franceschini et al. (2006; ellipticals: red down triangles and global samples: open squares); Pozzetti et al. (2007; open circles); Abraham et al. (2007; ellipticals: red up triangles). Local values are from Kochanek et al. (2001) for morphologically selected ellipticals (red star) and spirals (blue star) and whole sample (black star); Cole et al. (2001; circle) and Driver et al. (2006; colored rectangles). For clarity we do not show errorbars for other surveys.

to reproduce the observed trend at low and high redshifts. It predicts an increase in luminosity density by a factor 1.6 to 2.2 , up to $z \sim 1.2$, while the observations suggest a modest increase by a factor ranging from 1.0 to 1.4. The disagreement with the PLE model at highest redshift is even more pronounced than for the active sample suggesting that the number density of the quiescent galaxies must drop even faster.

\section{The stellar mass density up to $z=2$}

We now derive the stellar mass density, $\rho_{\star}$, up to $z=2$. To that end we convert our $K$-band luminosity densities (Table 1 ), to stellar mass densities via the relation $\rho_{\star}(z)=\rho_{L_{K}}(z) \times\left\langle M / L_{K}\right\rangle(z)$ and using the mass to light ratio equations determined in Sect. 4.

Our measurements of $\rho_{\star}$ for the three samples are shown in Fig. 13. The errorbars account for Poisson, photo- $z$ and cosmic variance uncertainties (as for $\rho_{L}$ ) and an additional uncertainty of 0.05 dex from $M / L$ estimates (assuming that the paramaters $a$ and $b$ in the $M / L$ relation are un-correlated).

\subsection{The evolution of the stellar mass density}

When moving back in time, the global population shows a small but regular decline up to $z \sim 1.1$ which accelerates at higher $z$.
By comparing with local estimates, we find that the total stellar mass has decreased by roughly a factor of $\sim 1.5,2$ and 4 up to $z \sim$ $1.0,1.5,2.0$. The compilation of previous surveys shows a large scatter by roughly a factor two and our estimates are located in the upper envelope. While the scatter can be in part due to mass estimates, for example the use or not of near IR data, the cosmic variance is most likely to be the dominant factor as discussed here and by Bell et al. (2003).

We quantify the stellar mass evolution of the active and quiescent samples with a simple linear fit with redshift up to $z=1.2$. Including local measurements, we get: $\Delta \log \rho_{\star}^{\text {Active }}=-0.05( \pm 0.09) z+8.51( \pm 0.04)$ and $\Delta \log \rho_{\star}^{\text {Quiescent }}=$ $-0.31( \pm 0.07) z+8.38( \pm 0.02)$. The active population shows a modest evolution, consistent with no evolution with a mean value $\rho_{\star}^{\text {Active }}=10^{8.49 \pm 0.04}$. This constancy of the active (blue) sequence has been pointed out by Borch et al. (2006) and by Martin et al. (2007) who derived a similar value. On the other hand, we observe that the stellar mass of the quiescent galaxies, $\rho_{\star}^{\text {Quiescent }}$, has increased by a factor $2 \pm 0.3$ between $z=1.2$ and $z=0$. Between $z=2$ and $z=1.2$ the evolution in stellar mass is even stronger, it increases by a factor of $\sim 10$. This suggests two different regimes in the build-up of the quiescent population. 


\subsection{Comparison to the integrated star formation rate estimates}

Thanks to the recent constraints on the cosmic star formation rate, we can compare the observed stellar mass density $\left(\rho_{\star}\right)$ to the predictions for the cosmic star formation rate $\left(\dot{\rho}_{\star}\right)$ over time, since the two quantities are simply related as follows:

$\rho_{\star}(t)=(1-R) \int_{0}^{t} \dot{\rho}_{\star}(t) \mathrm{d} t$

where $R$ is the recycling factor (fraction of stellar mass released in the interstellar medium) which is assumed, in the case of a Salpeter IMF, to be $R=0.3$ (Madau et al. 1998). For the SFR, $\dot{\rho}_{\star}(z)$, we use the uncorrected UV SFR as measured by Schiminovich et al. (2005) between $0 \leq z \leq 3$ that we have extended up to $z \sim 6$, based on measurements from Steidel et al. (1999), Bowens et al. (2006) and Bunker et al. (2004). To derive the dust corrected UV-SFR, we adopt a range of dust attenuation that varies between $1.1 \leq A_{\mathrm{FUV}} \leq 1.7$ which is consistent with local estimates (Buat et al. 2005) and with other dust-free SFR estimators up to $z \sim 1$ (Schiminovich et al. 2005; Hopkins \& Beacom 2006). We also use the SFR estimate from the analysis of Hopkins \& Beacom (2006) who compile most of the recent surveys from the UV to the Far-Infrared to derive a global, dust corrected SFR ${ }^{2}$. The two SFR histories are shown in an inset in Fig. 13 and are in good agreement within the range of dust attenuations we have considered. The result of integrating $\dot{\rho}_{\star}$ is shown as a shaded area. We emphasize that we did not impose beforehand that our integrated SFR be consistent with the stellar mass density at $z=0$. Nevertheless, it provides a remarkable fit in the redshift range probed in this work and encloses well the local estimates (see also Rudnick et al. 2006).

We find that our measurements are well represented by an attenuation value ranging from $1.1 \leq A_{\mathrm{FUV}} \leq 1.3$ ) between $0 \leq z \leq 1.5$. This constancy of the mean dust attenuation up to $z \sim 1.5$, can be qualitatively interpreted as a trade-off between two competing factors: - 1- star-forming galaxies at high- $z$ have less dust attenuation than their local counterparts at the same total luminosity (Reddy et al. 2006; Xu et al. 2007) -2star-forming galaxies at high- $z$ are intrinsically more luminuous (Arnouts et al. 2005; Le Floch et al. 2006). We can quantify these effects by looking at the relations between the $\left(L_{\text {Dust }} / L_{\mathrm{UV}}\right)$ ratio and the total luminosity $\left(L_{\mathrm{TOT}}=L_{\text {Dust }}+L_{\mathrm{UV}}\right)$, all quantities expressed as $v L_{v}$. From the local relation (Martin et al. 2005), a typical $L_{\mathrm{UV}}^{\star}(z=0)$ galaxy has a $L_{\text {Dust }} / L_{\mathrm{UV}} \sim 3.75$, corresponding to $A_{\mathrm{FUV}} \sim 1.3$ (Buat et al. 2005). If we let this galaxy evolve according to the evolution of the luminosity functions $\left(L_{\mathrm{FUV}} \sim(1+z)^{2.5}\right.$; Arnouts et al. 2005 and $L_{\mathrm{FIR}} \sim(1+z)^{3.2}$; Le Floch et al. 2006), at $z \sim 1.35$ we obtain a typical luminosity of $\log \left(L_{\mathrm{TOT}} / L_{\odot}\right) \sim 11.34$. We can finally estimate the amount of dust attenuation for this galaxy based on the relation from Reddy et al. (2006, their Eq. (5)) that holds in the redshift range $1 \leq z \leq 3$. We obtain $L_{\text {Dust }} / L_{\mathrm{UV}} \sim 4.3$, corresponding to $A_{\mathrm{FUV}} \sim 1.4$. This rough estimate shows that a typical $L^{\star}(z)$ galaxy that contributes to the bulk of the SFR at all $z$ shows, on average, a small evolution of the amount of dust attenuation, in agreement with the observation of the integrated dust corrected SFR in Fig. 13.

To summarize, with simplified but not unrealistic estimates of the dust correction, we observe that the two complementary quantities, the star formation rate and the stellar mass density,

\footnotetext{
${ }^{2}$ We adopt their SFR based on the modified Salpeter IMF which is converted to a Salpeter IMF by applying +0.2 dex in stellar mass.
}

are remarkably consistent with each other. This result is encouraging, and may provide some more fundamental informations about the initial mass function (IMF). The observations of the two quantities explore two different parts of the IMF (dominated by massive stars, a few $M_{\odot}$, for the SFR, and low mass stars, for stellar mass) and are extrapolated through the adopted IMF. The good agreement of these complementary observations over the last $10 \mathrm{Gyr}$, is a good support for an IMF that is on average universal, as already pointed out in previous work (e.g. Franceschini et al. 2006). We note that this remark is not specific to the Salpeter IMF adopted throughout this paper, but holds also for other IMFs suggested in the literature, as long as the quantities $\left(\rho_{\star}, \dot{\rho}_{\star}\right)$ are appropriately corrected for IMF effects.

\section{Discussion and conclusions}

In this work, we have used a unique large sample of 21200 galaxies selected at $3.6 \mu \mathrm{m}$ (based on the VVDS and SWIRE surveys) to investigate the evolution of the luminosity functions, luminosity densities, stellar mass to light relations and stellar mass densities up to $z \sim 2$. We have separated the active and quiescent galaxies based on an SED fitting procedure. We define as quiescent those galaxies which are best fit by an elliptical template. We have shown that this sample reproduces well the red sequence of the color bimodality $\left(N U V-r^{\prime}\right)$ (known to be a good separator between active and quiescent galaxies with $b \leq 0.1)$ and at the same time minimizes the contamination by dusty starbursts. We find that the active and quiescent populations follow different behaviours. In particular, there is a clear transition between two regimes in the evolution of the quiescent population at a redshift of $z=1.2$ (i.e. 8 Gyr ago).

\subsection{The last $8 \mathrm{Gyr}$}

The active population appears to be in place at $z \sim 1.2$ with a small evolution over this time laps except the aging of its stellar population. In contrast the quiescent sequence shows a gradual increase with a doubling of its stellar mass in line with previous optical studies (Bell et al. 2003; Faber et al. 2006; Brown et al. (2006).

The lack of evolution of the stellar mass density for the active, $\rho_{\star}^{\text {active }}$, and the doubling for quiescent, $\rho_{\star}^{\text {quiescent }}$, is surprising. The gradual increase of the global stellar mass density, over the last 8 Gyr can be easily explained by the formation of new stars by star forming galaxies at a rate described by the cosmic star formation rate. Or, similarly, it is found that intermediate mass galaxies $\left(M / M_{\odot} \sim 10.5\right)$ have specific SFRs allowing them to increase their stellar mass by a factor two since $z=1$ (Juneau et al. 2005). On the other hand, the increase of $\rho_{\star}^{\text {quiescent }}$ is in apparent contradiction with the definition of quiescent galaxies that cannot produce more than $5 \%$ of additional stellar mass over the last $8 \mathrm{Gyr}$ (due to the absence of star formation). The most plausible explanation for the increase of $\rho_{\star}^{\text {quiescent }}$ is a progressive migration of galaxies from the active to the quiescent population at a similar rate than that with which new stars are formed.

We quantify this evolution by a rough estimate of the stellar mass growth per unit of time defined as:

$\dot{\rho}_{X}=\frac{\rho_{\star}^{X}\left(z_{l}\right)-\rho_{\star}^{X}\left(z_{h}\right)}{t_{\text {univ }}\left(z_{l}\right)-t_{\text {univ }}\left(z_{h}\right)}$,

where $X$ refers to stellar mass of the considered sample in the redshift range $z_{l} \leq z \leq z_{h}$. We find that the stellar 
mass growth of the active population evolves very little with $\dot{\rho}_{\text {Active }}=0.005 \pm 0.003 M_{\odot} / \mathrm{Mpc}^{3} / \mathrm{yr}$, while the quiescent population has a much higher stellar mass growth with $\dot{\rho}_{\text {Quiescent }}=$ $0.017 \pm 0.004 M_{\odot} / \mathrm{Mpc}^{3} / \mathrm{yr}$. We can compare this value with the mass growth expected by integrating the star formation history over the same period of time. We find that $\dot{\rho}_{S F R}=$ $0.025 \pm 0.003 M_{\odot} / \mathrm{Mpc}^{3} / \mathrm{yr}$ (assuming $1.1 \leq A_{\mathrm{FUV}} \leq 1.3$ ). Under the assumption that the quiescent population has negligible residual star formation, its mass growth can be attributed to the mass flux of active galaxies moving into a quiescent mode $\left(\dot{\rho}_{A \rightarrow Q}=\dot{\rho}_{\text {Quiescent }}\right)$, and which appear to account for most of the global stellar mass growth derived from the SFR.

Additional evidence for this scenario is also presented by Vergani et al. (2007) who split the VVDS spectroscopic sample in active and quiescent galaxies based on $4000 \AA$ break. In order to satisfy the constraints from the stellar mass functions split in active and quiescent galaxies, they find that the star formation in some massive blue galaxies must have been quenched, moving these galaxies into the "red sequence".

\subsection{The major epoch of build-up for quiescent galaxies:}

The present analysis allows us to extend previous work to the redshift range between $2 \geq z \geq 1.2$ (3.2 Gyr $\leq T_{\text {univ }} \leq 5 \mathrm{Gyr}$ ). This appears to be a transition epoch, as evidenced by the strong increase in stellar mass, by a factor of $\sim 10$, of the quiescent population between $2 \geq z \geq 1.2$, while the active population increases still by a factor $\sim 2.5$. This translates into a stellar mass growth for the quiescent populations of $\sim 0.065 M_{\odot} / \mathrm{Mpc}^{3} / \mathrm{yr}$, which is more than 3 times faster than the evolution at $z \leq 1.2$. In contrast to $z \leq 1.2$, where most of the galaxies seem to be in place, at $z \geq 1.2$, galaxies are still in an active phase of mass assembly. In particular, the evolution of the quiescent population suggests that we are observing the epoch when, for the first time in the history of the universe, a large number of active galaxies are ending their star formation activity and start to build up a quiescent population. While the mechanism acting in this process is not clear, gas exhaustion, merging or other effects, this build-up happens a few Gyr after the peak of the cosmic SFR (Hopkins \& Beacom 2006). An interesting related information is the similar evolution followed by morphologically selected elliptical galaxies (Franceschini et al. 2006; Abraham et al. 2007). If not by chance, this coincidence could suggest that the build-up of the quiescent sequence is closely followed or preceded by a morphological transformation.

Acknowledgements. We are grateful to the referee for his careful reading of the manuscript and his suggestions. S.A. wants to thank B. Milliard for useful discussions. This work has been partially supported by the CNRS-INSU and the "Progamme National de Cosmologie" (France), the "Programme National Galaxies" (France) and a grant awarded for "EFIGI project" (grant \# 45500) from the French research ministry. C.J.W. is supported by the MAGPOP Marie Curie EU Research and Training Network.

\section{References}

Abraham, R. G., McCarthy, P. J., Glazebrook, K., et al. 2007 [arXiv:astroph/0701779]

Arnouts, S. 2003, in Multiwavelength Cosmology, proceeding, Mykonos, June 2003

Arnouts, S., Schiminovich, D., Ilbert, O., et al. 2005, ApJ, 619, L43

Baldry, I. K., Glazebrook, K., Brinchmann, J., et al. 2004, ApJ, 600, 681

Bell, E., \& de Jong, R. S. 2001, ApJ, 550, 212

Bell, E. F., McIntosh, D. H., Katz, N., \& Weinberg, M. D. 2003, ApJ, 585, 117

Bell, E., Wolf, C., Meisenheimer, K., et al. 2004, ApJ, 608, 752

Bell, E. F., Papovich, C., Wolf, C., et al. 2005, ApJ, 625, 23
Benson, A. J., Bower, R. G., Frenk, C. S., et al. 2003, ApJ, 599, 38 Bertin, E., \& Arnouts, S. 1996, A\&AS, 117, 393

Bolzonnella, M., Miralles, J. M., \& Pello, R. 2000, A\&A, 363, 476

Borch, A., Meisenheimer, K., Bell, E., et al. 2006, A\&A, 453, 869

Bouwens, R. J., Illingworth, G. D., Blakeslee, J. P., \& Franx, M. 2006, ApJ, 653, 53

Brinchmann, J., \& Ellis, R. S. 2000, ApJ, 536, L77

Brown, M. J. I., Dey, A., Jannuzi, B. T., et al. 2007, ApJ, 654, 858

Bruzual, G., \& Charlot, S. 2003, MNRAS, 344, 1000

Buat, V., Iglesias-Paramo, J., Seibert, M., et al. 2005, ApJ, 619, L51

Bundy, K., Ellis, R. S., \& Conselice, C. J. 2005, ApJ, 625, 621

Bundy, K., Ellis, R. S., Conselice, C. J., et al. 2006, ApJ, 651, 120

Bunker, A. J., Stanway, E. R., Ellis, R. S., \& Mc Mahon, R. G. 2004, MNRAS, 355,374

Caputi, K. I., Dunlop, J. S., Mc Lure, R. J., \& Roche, N. D. 2005, MNRAS, 361, 607

Chabrier, G. 2003, PASP, 115, 763

Cimatti, A., Mignoli, M., Daddi, E., et al. 2002, A\&A, 392, 395

Cimatti, A., Daddi, E., Renzini, A., et al. 2004, Nature, 430, 184

Cimatti, A., Daddi, E., \& Renzini, A. 2006, A\&A, 453, 29

Cirasuolo, M., McLure, R. J., Dunlop, J. S., et al. 2006 [arXiv:astro-ph/0609287]

Cohen, J. G. 2002, ApJ, 567, 672

Cole, S., Norberg, P., \& Baugh, C. M. 2001, MNRAS, 326, 255

Cowie, L. L., Songaila, A., Hu, E., \& Cohen, J. G. 1996, AJ, 112, 839

Croton, D. J., Springel, V., White, S. D. M., et al. 2006, MNRAS, 365, 11

Daddi, E., Cimatti, E., Broadhurst, T., et al. 2002, A\&A, 384, 1

Daddi, E., Cimatti, E., Renzini, A., et al. 2004, ApJ, 617, 746

de Lucia, G., Springel, V., White, S. D. M., et al. 2006, MNRAS, 366, 499

Dickinson, M., Papovich, C., Ferguson, H. C., et al. 2003, ApJ, 587, 25

Driver, S. P., Allen, P. D., Graham, A. W., et al. 2006, MNRAS, 368, 414

Drory, N., Bender, R., Feulner, G., et al. 2003, ApJ, 595, 698

Drory, N., Bender, R., Feulner, G., et al. 2004, ApJ, 608, 742

Drory, N., Salvato, M., Gabash, A., et al. 2005, ApJ, 619, L131

Faber, S. M., Willmer, C. N. A., Wolf, C., et al. 2005 [arXiv: astro-ph/0506044]

Fazio, G. G., Hora, J. L., \& Allen, L. E. 2004, ApJS, 154, 39

Fioc, M., \& Rocca-Volmerange, B. 1997, A\&A, 326, 950

Fontana, A., Donnarumma, I., Vanzella, E., et al. 2003, ApJ, 594, 9

Fontana, A., Pozzetti, L., Donnarumma, I., et al. 2004, A\&A, 424, 23

Fontana, A., Salimbeni, S., Grazian, A., et al. 2006, A\&A, 459, 745

Franceschini, A., Rodighiero, G., \& Cassata, P. 2006, A\&A, 453, 397

Franzetti, P., Scodeggio, M., Garilli, B., et al. 2007, A\&A, 465, 711

Gwyn, S. D. J., \& Hartwick, F. D. A. 2005, AJ, 130, 1337

Heavens, A., Panter, B., Jimenez, R., \& Dunlop, J. 2004, Nature, 428, 625

Heinis, S., Milliard, B., Arnouts, S., et al. 2007, ApJ, accepted [arXiv:0706.1076]

Hopkins, A., \& Beacom, J. F. 2006, ApJ, 651, 142

Ilbert, O., Tresse, L., Arnouts, S., et al. 2004, MNRAS, 351, 541

Ilbert, O., Arnouts, S., McCracken, H. J., et al. 2006a, A\&A, 457, 841

Ilbert, O., Lauger, S., Tresse, L., et al. 2006b, A\&A, 453, 809

Juneau, S., Glazebrook, K., Crampton, D., et al. 2005, ApJ, 619, L135

Kauffmann, G., Heckman, T. M., White, S. D. M., et al. 2003, MNRAS, 341, 33

Kochanek, C. S., Pahre, M. A., \& Falco, E. E. 2001, ApJ, 560, 566

Lawrence, A., Warren, S. J., Almaini, O., et al. 2006 [arXiv: astro-ph/0604426]

Le Fèvre, O., Vettolani, G., \& Garilli, B. 2005, 439, 845

Lilly, S. J., Le Fèvre, O., Hammer, F., \& Crampton, D. 1996, ApJ, 460, L1

Lonsdale, C. J., Smith, H. E., Rowan-Robinson, M., et al. 2003, PASP, 115, 897

Madau, P., Pozzetti, L., \& Dickinson, M. 1998, ApJ, 498, 106

Madgwick, D. S., Lahav, O., Baldry, I. K., et al. 2002, MNRAS, 333, 133

Madgwick, D. S., Somerville, R., Lahav, O., \& Ellis, R. 2003, MNRAS, 343, 871

Maraston, C., Daddi, E., Renzini, A., et al. 2006, ApJ, 652, 85

Marinoni, C., Le Fèvre, O., Meneux, B., et al. 2005, A\&A, 442, 801

Martin, C. D., Fanson, J., Schiminovich, D., et al. 2005, ApJ, 619, L1

Martin, C. D., Wyder, T. K., Schiminovich, D., et al. 2007, ApJ, accepted [arXiv: astro-ph/0703281]

Mo, H. J., \& White, S. D. M. 1996, MNRAS, 282, 347

Nagamine, K., Cen, R., \& Ostriker, J. P. 2001, Birth and Evolution of the Universe, 383

Pozzetti, et al. 2007, A\&A, submitted

Pozzetti, L., \& Mannucci, F. 2000, MNRAS, 317, L17

Reddy, N. A., Steidel, C. C., Fadda, D., et al. 2006, ApJ, 644, 792

Rudnick, G., Labbé, I., Forster Schreiber, N. M., et al. 2006, ApJ, 650, 624

Saracco, P., Fiano, A., Chincarini, G., et al. 2006, MNRAS, 367, 349

Salim, S., Charlot, S., Rich, M. R., et al. 2005, ApJ, 619, L39

Schiminovich, D., Ilbert, O., Arnouts, S., et al. 2005, ApJ, 619, L47 
Somerville, R., Lee, K., Ferguson, H. C., et al. 2004, ApJ, 600, 171 Springel, V., Frenk, C. S., \& White, S. D. M. 2006, Nature, 440, 1137 Steidel, C. C., Adelberger, K. L., \& Giavalisco, M. 1999, ApJ, 519, 1 Surace, J. A., Shupe, D. L., Fang, F., et al. 2005, AAS, 207.6301

Takeuchi, T. T., Buat, V., \& Burgarella, D. 2005, A\&A, 440, L17

Treu, T., Ellis, R. S., Liao, T. X., et al. 2005, ApJ, 633, 174

van Dokkum, P. G. 2005, AJ, 130, 2647

van Dokkum, P. G., \& Stanford, S. A. 2003, ApJ, 585, 78

Warren, S. J., Hambly, N. C., Dye, S., et al. 2007, MNRAS, 375, 213

Wyder, T., Martin, C. D., \& Schiminovich, D. 2007, ApJ, accepted [arXiv:0706.3938]

Xu, C. K., Shupe, D., Buat, V., et al. 2007 [arXiv:astro-ph/0701737]

Yoshikawa, K., Taruya, A., Jing, Y. P., \& Suto, Y. 2001, ApJ, 558, 520

Zucca, E., Ilbert, O., Bardelli, S., et al. 2006, A\&A, 455, 879

1 Canada-France-Hawaii Telescope Corporation, Kamuela, HI-96743, USA

2 Laboratoire d'Astrophysique de Marseille, UMR 6110, BP 8, 13376 Marseille Cedex 12, France e-mail: arnouts@cfht.hawaii.edu

3 INAF-Osservatorio Astronomico di Bologna, via Ranzani 1, 40127 Bologna, Italy
4 Institute for Astronomy, 2680 Woodlawn Dr., University of Hawaii, Honolulu 96822, Hawaii

5 Institut d'Astrophysique de Paris, UMR 7095, 98 bis Bvd Arago, 75014 Paris, France

${ }^{6}$ Laboratoire d'Astrophysique de Toulouse, UMR 5572, 14 av. E. Belin, 31400 Toulouse, France

7 INAF-Osservatorio Astronomico di Brera, via Brera 28, Milan, Italy

8 University of California, San Diego 9500 Gilman Dr. La Jolla, CA 92093-0424, USA

9 Spitzer Science Center, Mail Stop 314-6, 1200 East California Boulevard, Pasadena, CA 91125, USA

${ }_{10}$ IASF-INAF, via Bassini 15, 20133 Milano, Italy

11 INAF-Osservatorio Astronomico di Capodimonte, via Moiariello 16, 80131 Napoli, Italy

12 Geneva Observatory, ch. des Maillettes 51, 1290 Sauverny, Switzerland

13 Centre de Physique Théorique, UMR 6207 CNRS-Université de Provence, 13288 Marseille, France

14 School of Physics \& Astronomy, University of Nottingham, University Park, Nottingham, NG72RD, UK

15 Astrophysical Institute Potsdam, An der Sternwarte 16, 14482 Potsdam, Germany 